Engineering and Computational Mechanics Volume 170 Issue EM2

One-dimensional and two-dimensional Green-Naghdi equations for sloshing in shallow basins

Jalali and Borthwick
Proceedings of the Institution of Civil Engineers

Engineering and Computational Mechanics 170 June 2017 Issue EM2 Pages 49-70 http://dx.doi.org/10.1680/jencm.16.00021 Paper 1600021

Received 08/06/2016

Published online 24/02/2017

Accepted 16/01/2017

Keywords: computational mechanics/hydraulics

\& hydrodynamics/mathematical modelling

Published with permission by the ICE under the CC-BY license.

(http://creativecommons.org/licenses/by/4.0/)

\title{
One-dimensional and two-dimensional Green-Naghdi equations for sloshing in shallow basins
}

Mohammad R. Jalali BSc, MSc, PhD

PhD student, Institute for Energy Systems, The University of Edinburgh, Edinburgh, UK (corresponding author: reza.jalali@ed.ac.uk)
Alistair Borthwick PhD, DSc, CEng, FICE, FREng, FRSE

Professor, Institute for Energy Systems, The University of Edinburgh, Edinburgh, UK

This paper presents a verified model of weakly non-linear wave sloshing in shallow basins, based on level I Green-Naghdi (GN) mass and momentum equations derived for mild-sloped beds. The model is verified for sloshing of an initially sinusoidal free surface perturbation in a square tank with a horizontal bed. The model is also used to investigate free surface sloshing of an initial Gaussian hump in closed square basins, over horizontal and nonuniform bed topographies. Analysis of the free surface slosh motions demonstrates that the model gives predictions in satisfactory agreement with the analytical solution of linearised shallow water theory obtained by Lamb. Discrepancies between GN predictions and linear analytical solutions arise from the effect of wave non-linearities arising from the wave amplitude itself and wave-wave interactions.

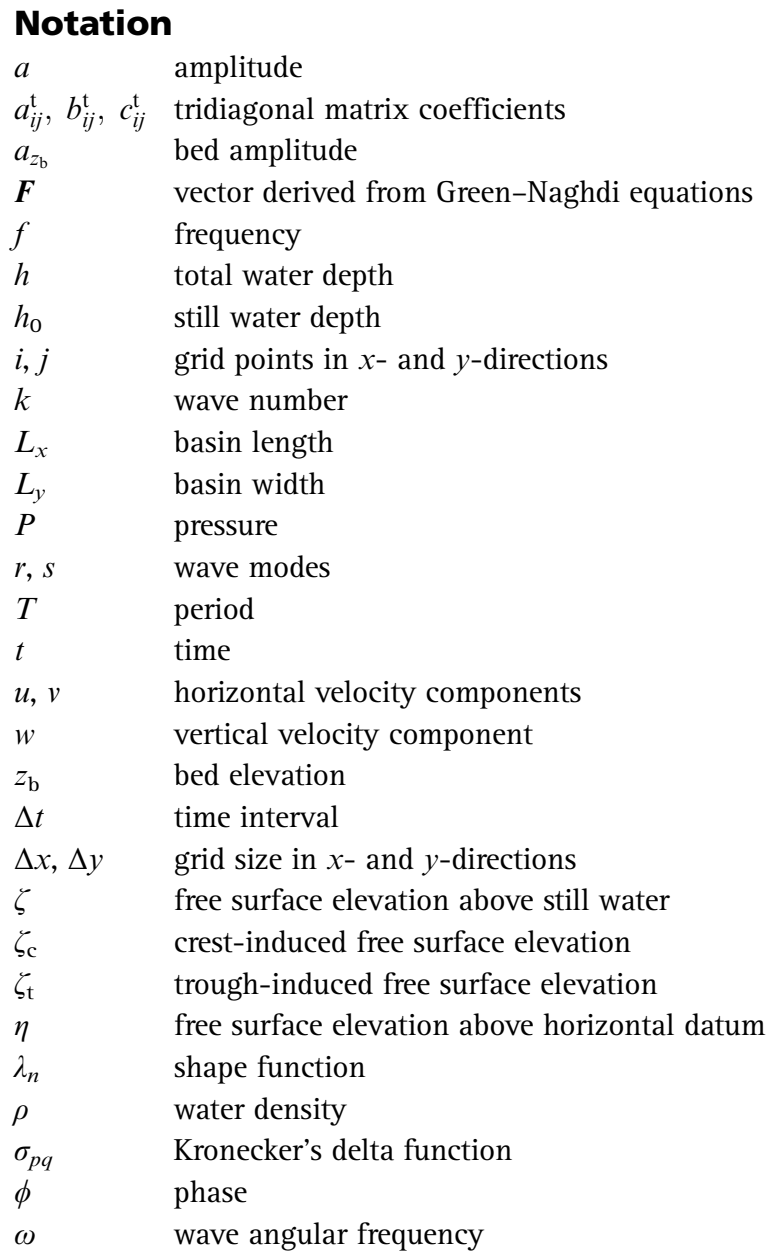

\section{Introduction}

Slosh motions occur in liquid ballast tanks of ships, liquefied natural gas tankers and containers subject to seismic excitation. The response of the liquid free surface depends on the amplitude of the initial disturbance and the natural frequencies of motion, and involves complex fluid-structure interactions (Ibrahim, 2005). In practice, civil engineers may use sloshing tests to assess the likelihood of such phenomena occurring in oil tanks, elevated water towers and in reservoirs. According to Sarpkaya and Isaacson (1981) the earliest theories of progressive and sloshing waves were developed by Airy and Stokes, which were based on potential theory idealisations. Airy's theory was linear, so that it was strictly derived for waves of zero amplitude. Stokes extended the theory to deal with waves of finite amplitude, and he obtained series solutions that were later computed to high order by many hydrodynamicists in the late twentieth century (for more details see Sarpkaya and Isaacson (1981)). To extend to more realistic domains, computational methods have become widely used. These include: (a) boundary-element and finite-element potential flow solvers; (b) computational fluid dynamics-Navier-Stokes solvers with volume of fluid treatment of the free surface, Navier-Stokes solvers with level-set treatment of the free surface, NavierStokes solvers with mappings of the free surface; and (c) smoothed-particle hydrodynamics. The above-mentioned three-dimensional (3D) computational methods undergo inherently high computational expense; therefore, considerable effort has gone into depth-averaged approaches, they being cheaper to compute and yet capturing much of the physics. Examples of such approaches include shallow water equations 
(SWEs) (e.g. Lamb, 1916), and more recently Green-Naghdi (GN) equations. Non-linear SWEs are the depth-averaged form of continuity and Navier-Stokes momentum equations. Since SWEs neglect vertical motions and the consequent hydrostatic pressure, these equations are usually restricted to long-wave behaviour. Thus, SWEs are limited to shallow depth (surf zone) of the ocean (Bonneton et al., 2011; Nadiga et al., 1996). Green and Naghdi (1976) pioneered the development of non-linear equations for two-dimensional (2D) incompressible inviscid fluid sheets. Green and Naghdi (1976) proposed a theory of fluid sheets known as GN theory to model the 2D continuum of unsteady inviscid 3D flows. The theory facilitated prediction of unsteady, non-periodic, free surface flows. GN theory utilises some aspects of perturbation analysis in building up first-, second- and higher-order approximations (called levels) to layer-averaged mass and momentum equations. According to Webster and Shields (1991), the GN approach assumes a particular flow kinematic structure in the vertical direction for shallow-water problems. The fluid velocity profile is a finite sum of coefficients depending on space and time multiplied by a weighting function. GN fluid sheet theory reduces the dimensions from three to two, yielding equations that can be solved efficiently so that no scale is introduced and no term is deleted (Webster and Shields, 1991). Nevertheless, the lowest level of GN theory permits the kinematic boundary conditions to be satisfied. There are two types of GN theory: restricted and unrestricted. The former successfully models irrotational shallow-water flow field. Restricted GN theory was derived from the first level of the direct theory by means of a constrained director (Shields and Webster, 1988). Later, this procedure was extended to the $k$ th level theory (Demirbilek and Webster, 1992). In other words, in a restricted GN theory, the $k$ components of the 2D velocity components are constrained. Demirbilek and Webster (1992) developed an unrestricted version of GN theory of shallow water by enforcing conservation of mass and momentum in the vertical direction and implementing exact boundary conditions. They demonstrated that GN theory can appropriately predict the behaviour of a non-linear numerical wave tank. According to Webster and Shields (1991), GN sheet theory is placed between classical perturbation methods and pure numerical schemes. Webster and Shields (1991) note that for classical perturbation methods, there is usually no evidence that the assumed series is convergent. However, in certain flow problems, such as $2 \mathrm{D}$ water waves in both shallow and deep water addressed by GN theory, there is ample evidence of convergence. There is another difference between classical perturbation methods and GN theory. The former exactly satisfies field equations but partially satisfies boundary conditions. Thus, the classical perturbation methods show inconsistencies, while the GN theory is self-consistent since the boundary conditions are exactly satisfied, when the field equations are partially approximated. With regard to coastal engineering, the present GN model is merely applicable to the shallow depth (before the surf zone) and the intermediate depth of ocean (Jalali, 2016). GN levels higher than I can be used for the deep water ocean (Webster and Shields, 1991). The present paper describes the application of $2 \mathrm{D}$ and one-dimensional (1D) level I GN equations (according to Jalali (2016)) to free surface sloshing in closed square basins, with horizontal and non-uniform bed topographies. Sections 2 and 3 present the derivation of governing equations. Sections 4 , 5 and 6 outline the numerical implementation. Section 7 presents the results for initial sinusoidal and Gaussian free surface perturbations. Section 8 is the summary of the main findings.

\section{Derivation of continuity equation of level I GN equation}

In a 3D Cartesian system, the classical mass conservation equation is applied to drive the GN continuity equation

1. $\frac{\partial u}{\partial x}+\frac{\partial v}{\partial y}+\frac{\partial w}{\partial z}=0$

In which, $u, v$ and $w$ are the velocity components in $x, y$ and $z$ directions. In the present derivation of GN equations, the total depth, $h$, is $h=h_{0}+\zeta$. Here $h_{0}$ is the still water depth and $\zeta$ is the free surface elevation above still water level. The elevation of free surface above the fixed horizontal datum, $\eta$, is $\eta=h+z_{\mathrm{b}}$. Here, $z_{\mathrm{b}}$ is the bed elevation above a fixed horizontal datum. The kinematic bed and free surface boundary condition are as follows

2. $\left.\quad d\right|_{\mathrm{b}}=\frac{\partial z_{\mathrm{b}}}{\partial t}+u \frac{\partial z_{\mathrm{b}}}{\partial x}+v \frac{\partial z_{\mathrm{b}}}{\partial y}$

3. $\left.\quad d\right|_{\mathrm{s}}=\frac{\partial \eta}{\partial t}+u \frac{\partial \eta}{\partial x}+v \frac{\partial \eta}{\partial y}$

where $\left.d\right|_{\mathrm{b}}$ is the kinematic bed boundary condition and $\left.d\right|_{\mathrm{s}}$ is the kinematic free surface boundary condition. To derive the GN continuity equation, it is assumed that the velocity vector, $\boldsymbol{V}$, can be written as follows

4. $\quad \boldsymbol{V}(x, y, z, t)=\sum_{n=0}^{e} \boldsymbol{W}_{n}(x, y, t) \lambda_{n}(z)$

where $\boldsymbol{W}_{n}=\left(u_{n}, v_{n}, w_{n}\right)$ is a vector of velocity component approximations at level $n ; \lambda_{n}$ are the assumed shape functions depending on the $z$-direction and $e$ is the level of 
approximation of GN theory. Expansion of Equation 4 for level I gives the following velocity parameters

$$
\begin{aligned}
u(x, y, z, t) & =u_{0}(x, y, t) \\
v(x, y, z, t) & =v_{0}(x, y, t) \\
w(x, y, z, t) & =w_{0}(x, y, t)+w_{1}(x, y, z, t)\left(z-z_{\mathrm{b}}\right)
\end{aligned}
$$

In which

$$
\lambda_{0 x}=\lambda_{0 y}=\lambda_{0 z}=1, \quad \lambda_{1 x}=\lambda_{1 y}=\lambda_{1 z}=\left(z-z_{\mathrm{b}}\right)
$$

and

$u_{1}(x, y, t)=v_{1}(x, y, t)=0$ (for more details see Demirbilek and Webster (1992)).

By applying Equation 5 in Equations 1 and 2, the values of $w_{1}$ and $w_{0}$ are obtained

6. $w_{1}=-\left(\frac{\partial u_{0}}{\partial x}+\frac{\partial v_{0}}{\partial y}\right)$

7. $w_{0}=\frac{\partial z_{\mathrm{b}}}{\partial t}+u_{0} \frac{\partial z_{\mathrm{b}}}{\partial x}+v_{0} \frac{\partial z_{\mathrm{b}}}{\partial y}$

By using Equation 5 in Equation 3 and replacing Equations 6 and 7 into the resulted equation, the 2D level I GN continuity equation is derived

8. $\frac{\partial h}{\partial t}+\frac{\partial u_{0} h}{\partial x}+\frac{\partial v_{0} h}{\partial y}=0$

in which $h$ is the total depth and $\left(u_{0}, v_{0}\right)$ are the horizontal velocity components at a particular point $(x, y)$ and time $t$.

\section{Derivation of $x$-direction momentum of level I GN equation}

The general momentum conservation equation expanded in the $x$-direction is as follows

9. $\frac{\partial \rho u}{\partial t}+\frac{\partial \rho u u}{\partial x}+\frac{\partial \rho u v}{\partial y}+\frac{\partial \rho u w}{\partial z}=-\frac{\partial P}{\partial x}$

in which $\rho$ is the water density. Depth integrating Equation 9 and then using the chain rule for the fourth term and applying the Leibnitz rule for the right-hand side term result in

$$
\begin{aligned}
& \int_{z_{\mathrm{b}}}^{\eta} \frac{\partial \rho u}{\partial t} \lambda_{n} \mathrm{~d} z+\int_{z_{\mathrm{b}}}^{\eta} \frac{\partial \rho v}{\partial t} \lambda_{n} \mathrm{~d} z+\left.\rho u w \lambda_{n}\right|_{z_{\mathrm{b}}} ^{\eta}-\int_{z_{\mathrm{b}}}^{\eta} \rho u w \lambda_{n}^{\prime} \mathrm{d} z \\
& \quad=-\frac{\partial P_{n}}{\partial x}+\left.\hat{P} \frac{\partial \eta}{\partial x} \lambda_{n}\right|_{\eta}-\left.\bar{P} \frac{\partial z_{\mathrm{b}}}{\partial x} \lambda_{n}\right|_{z_{\mathrm{b}}}
\end{aligned}
$$

where $P_{n}=\partial\left(\int_{z_{\mathrm{b}}}^{\eta} P \lambda_{n} \mathrm{~d} z\right) / \partial x ; \hat{P}$ is pressure at the free surface (here $\hat{P}=0$ ) and $\bar{P}$ is the pressure at the bottom. Applying Equation 4 in Equation 10

$$
\begin{aligned}
& \sum_{m=0}^{e=1} \rho \frac{\partial u_{m}}{\partial t} y_{m n}+\sum_{m=0}^{e=1} \sum_{r=0}^{e=1} \rho \frac{\partial u_{m} u_{r}}{\partial x} y_{m r n} \\
& \quad+\sum_{m=0}^{e=1} \sum_{r=0}^{e=1} \rho \frac{\partial u_{m} v_{r}}{\partial y} y_{m r n}+\left.\sum_{m=0}^{e=1} \sum_{r=0}^{e=1} \rho u_{m} w_{r} \lambda_{m} \lambda_{r} \lambda_{n}\right|_{z_{\mathrm{b}}} ^{\eta} \\
& \quad-\sum_{m=0}^{e=1} \sum_{r=0}^{e=1} \rho u_{m} w_{r} y_{m r}^{n}=-\frac{\partial P_{n}}{\partial x}+\left.\hat{P} \frac{\partial \eta}{\partial x} \lambda_{n}\right|_{\eta}-\left.\bar{P} \frac{\partial z_{b}}{\partial x} \lambda_{n}\right|_{z_{\mathrm{b}}}
\end{aligned}
$$

where $\quad y_{m n}=\int_{z_{\mathrm{b}}}^{\eta} \lambda_{m} \lambda_{n} \mathrm{~d} z, y_{m r n}=\int_{z_{\mathrm{b}}}^{\eta} \lambda_{m} \lambda_{r} \lambda_{n} \mathrm{~d} z \quad$ and $\quad y_{m r}^{n}=$ $\int_{z_{\mathrm{b}}}^{\eta} \lambda_{m} \lambda_{r} \lambda_{n}{ }_{n} \mathrm{~d} z$ (for more details see chapter 2 of the $\mathrm{PhD}$ thesis by Jalali (2016) and chapter 4 of the PhD thesis by Haniffah (2013)).

Applying Equation 4 in Equation 1 and using the KrylovKantorovich method for the third term of the obtained equation result in

12. $\sum_{r=0}^{e=1} \frac{\partial u_{r}}{\partial x} \lambda_{r}+\sum_{r=0}^{e=1} \frac{\partial v_{r}}{\partial y} \lambda_{r}+\sum_{r=0}^{e=1} w_{r} \lambda_{n}^{\prime}=0$

Here, the index $n$ changes to $r$ in Equation 4 .

By summing over $m$ and then depth integrating, Equation 12 becomes

13. $\sum_{m=0}^{e=1} \sum_{r=0}^{e=1} \frac{\partial u_{r}}{\partial x} y_{m n r}=-\sum_{m=0}^{e=1} \sum_{r=0}^{e=1} \frac{\partial v_{r}}{\partial y} y_{m r n}-\sum_{m=0}^{e=1} \sum_{r=0}^{e=1} w_{r} y_{m n}^{r}$

By inserting Equation 13 into Equation 11 and implementing the chain rule for the second and third terms of Equation 11, the constrained $x$-direction momentum equation of level I GN equation is derived

$$
\begin{aligned}
& \sum_{m=0}^{e=1} \rho \frac{\partial u_{m}}{\partial t} y_{m n}+\sum_{m=0}^{e=1} \sum_{r=0}^{e=1} \rho \frac{\partial u_{m}}{\partial x} u_{r} y_{m r n} \\
& \quad+\sum_{m=0}^{e=1} \sum_{r=0}^{e=1} \rho \frac{\partial u_{m}}{\partial y} v_{r} y_{m r n}+\sum_{m=0}^{e=1} \sum_{r=0}^{e=1} \rho u_{m} w_{r} y_{n r}^{m} \\
& \quad=-\frac{\partial P_{n}}{\partial x}-\left.\bar{P} \frac{\partial z_{\mathrm{b}}}{\partial x} \lambda_{n}\right|_{z_{\mathrm{b}}}
\end{aligned}
$$


Derivation of the GN equation in $z$-direction is similar to the derivation of GN equation in $x$-direction; therefore, the detailed derivation is not included here for brevity. The constrained $z$-direction momentum equation of level I GN equation is

$$
\sum_{m=0}^{e=1} \rho \frac{\partial w_{m}}{\partial t} y_{m n}+\sum_{m=0}^{e=1} \sum_{r=0}^{e=1} \rho \frac{\partial w_{m}}{\partial x} u_{r} y_{m r n}
$$

15.

$$
\begin{aligned}
& +\sum_{m=0}^{e=1} \sum_{r=0}^{e=1} \rho \frac{\partial w_{m}}{\partial y} v_{r} y_{m r n}+\sum_{m=0}^{e=1} \sum_{r=0}^{e=1} \rho w_{m} w_{r} y_{r n}^{m} \\
& =\left.\bar{P} \lambda_{n}\right|_{z_{\mathrm{b}}}+P_{n}^{\prime}-\rho \boldsymbol{g} y_{n}
\end{aligned}
$$

where $P^{\prime}{ }_{n}=\int_{z_{\mathrm{b}}}^{\eta} P \lambda_{n}^{\prime} \mathrm{d} z$ and $\boldsymbol{g}$ is the gravitational acceleration.

(To learn more about the derivation of GN momentum equations in this paper, refer to the $\mathrm{PhD}$ theses by Haniffah (2013) and Jalali (2016).)

Equations 14 and 15 are expanded for level I $\left\{\left(u_{0}, u_{1}\right),\left(v_{0}, v_{1}\right)\right.$, $\left.\left(w_{0}, w_{1}\right)\right\}$. In $z$-momentum GN Equation $15, w_{0}$ and $w_{1}$ are replaced by Equations 6 and 7. Two sets of equations are derived for $x$ - and $z$-momentum GN equations $(n=0$, level 0 , and $n=1$, level I). In $z$-momentum GN Equation $15, P_{0}^{\prime}=0$ and $P_{1}^{\prime}=P_{0}$. For eliminating the effect of $P_{0}$ differentiation is applied with respect to $x$ for $n=1$ set of $z$-momentum GN equation and the obtained equation is added to the $n=0$ set of $x$-momentum GN equation. Moreover, the effect of the bottom pressure term, $\bar{P}$, is eliminated by multiplying $\left(\partial z_{\mathrm{b}} / \partial x\right)$ the $n=0$ set of $z$-momentum GN equation and adding the resulted equation to the $n=1$ set of $z$-momentum GN equation. Finally, the 2D level I $x$-momentum GN equation for the stationary bed, $\left(\partial z_{\mathrm{b}} / \partial t=\partial^{2} z_{\mathrm{b}} / \partial x \partial t=\partial^{2} z_{\mathrm{b}} / \partial y \partial t=0\right)$, and non-uniform bathymetry is derived
By simplifying Equation 16, the 2D level I $x$-momentum GN equation for uniform bathymetry is

$$
\begin{aligned}
& \boldsymbol{g} \frac{\partial \eta}{\partial x}+h\left(\frac{\partial h}{\partial x}\right)\left[-\frac{\partial^{2} u_{0}}{\partial x \partial t}-\frac{\partial^{2} v_{0}}{\partial y \partial t}-u_{0} \frac{\partial^{2} u_{0}}{\partial x^{2}}-v_{0} \frac{\partial^{2} v_{0}}{\partial y^{2}}\right. \\
& \left.-\frac{\partial^{2} u_{0} v_{0}}{\partial x \partial y}+\left(\frac{\partial u_{0}}{\partial x}+\frac{\partial v_{0}}{\partial y}\right)^{2}\right]+\frac{h^{2}}{3}\left[-\frac{\partial^{3} u_{0}}{\partial x^{2} \partial t}-\frac{\partial^{3} v_{0}}{\partial x \partial y \partial t}\right. \\
& -u_{0} \frac{\partial^{3} u_{0}}{\partial x^{3}}-\frac{\partial^{3} u_{0} v_{0}}{\partial x^{2} \partial y}-v_{0} \frac{\partial^{3} v_{0}}{\partial x \partial y^{2}}-\frac{\partial v_{0}}{\partial x}\left(\frac{\partial^{2} u_{0}}{\partial x \partial y}+\frac{\partial^{2} v_{0}}{\partial y^{2}}\right) \\
& \left.+\left(\frac{\partial u_{0}}{\partial x}+2 \frac{\partial v_{0}}{\partial y}\right)\left(\frac{\partial^{2} v_{0}}{\partial x \partial y}+\frac{\partial^{2} u_{0}}{\partial x^{2}}\right)\right] \\
& +\left(\frac{\partial u_{0}}{\partial t}+u_{0} \frac{\partial u_{0}}{\partial x}+v_{0} \frac{\partial u_{0}}{\partial y}\right)=0
\end{aligned}
$$

The 2D level I $y$-direction momentum GN equation and its derivation are not included here for brevity (for complete detailed derivations of level I GN equations, see the $\mathrm{PhD}$ theses by Haniffah (2013) and Jalali (2016)). The corresponding 1D level I $x$-momentum GN equation for uniform bathymetry is

$$
\begin{gathered}
\boldsymbol{g} \frac{\partial \eta}{\partial x}+h\left(\frac{\partial h}{\partial x}\right)\left[-\frac{\partial^{2} u_{0}}{\partial x \partial t}-u_{0} \frac{\partial^{2} u_{0}}{\partial x^{2}}+\left(\frac{\partial u_{0}}{\partial x}\right)^{2}\right] \\
+\frac{h^{2}}{3}\left[-\frac{\partial^{3} u_{0}}{\partial x^{2} \partial t}-u_{0} \frac{\partial^{3} u_{0}}{\partial x^{3}}+\frac{\partial u_{0}}{\partial x} \frac{\partial^{2} u_{0}}{\partial x^{2}}\right] \\
+\left(\frac{\partial u_{0}}{\partial t}+u_{0} \frac{\partial u_{0}}{\partial x}\right)=0
\end{gathered}
$$$$
18 .
$$

The corresponding 1D level I GN continuity equation is

19. $\frac{\partial h}{\partial t}+\frac{\partial u_{0} h}{\partial x}=0$

16.

$$
\begin{aligned}
& \frac{\partial \eta}{\partial x}\left[\frac{\partial z_{\mathrm{b}}}{\partial x}\left(\frac{\partial u_{0}}{\partial t}+v_{0} \frac{\partial u_{0}}{\partial y}-u_{0} \frac{\partial v_{0}}{\partial y}\right)+u_{0}^{2} \frac{\partial^{2} z_{\mathrm{b}}}{\partial x^{2}}+\frac{\partial z_{\mathrm{b}}}{\partial y}\left(\frac{\partial v_{0}}{\partial t}+u_{0} \frac{\partial v_{0}}{\partial x}-v_{0} \frac{\partial u_{0}}{\partial x}\right)+v_{0}^{2} \frac{\partial^{2} z_{\mathrm{b}}}{\partial y^{2}}+2 u_{0} v_{0} \frac{\partial^{2} z_{\mathrm{b}}}{\partial x \partial y}+\boldsymbol{g}\right] \\
& +\frac{h}{2}\left[\frac{\partial z_{\mathrm{b}}}{\partial x}\left(\frac{\partial^{2} u_{0}}{\partial x \partial t}+\frac{\partial v_{0}}{\partial x} \frac{\partial u_{0}}{\partial y}+v_{0} \frac{\partial^{2} u_{0}}{\partial x \partial y}-\frac{\partial u_{0}}{\partial x} \frac{\partial v_{0}}{\partial y}-u_{0} \frac{\partial^{2} v_{0}}{\partial x \partial y}\right)+\frac{\partial^{2} z_{\mathrm{b}}}{\partial x^{2}}\left(\frac{\partial u_{0}}{\partial t}+2 u_{0} \frac{\partial u_{0}}{\partial x}+v_{0} \frac{\partial u_{0}}{\partial y}-u_{0} \frac{\partial v_{0}}{\partial y}\right)\right. \\
& \left.+\frac{\partial z_{\mathrm{b}}}{\partial y}\left(\frac{\partial^{2} v_{0}}{\partial x \partial t}+u_{0} \frac{\partial^{2} v_{0}}{\partial x^{2}}-v_{0} \frac{\partial^{2} u_{0}}{\partial x^{2}}\right)+\frac{\partial^{2} z_{\mathrm{b}}}{\partial x \partial y}\left(\frac{\partial v_{0}}{\partial t}+3 u_{0} \frac{\partial v_{0}}{\partial x}+v_{0} \frac{\partial u_{0}}{\partial x}\right)+u_{0}^{2} \frac{\partial^{3} z_{\mathrm{b}}}{\partial x^{3}}+2 u_{0} v_{0} \frac{\partial^{3} z_{\mathrm{b}}}{\partial x^{2} \partial y}+2 v_{0} \frac{\partial v_{0}}{\partial x} \frac{\partial^{2} z_{\mathrm{b}}}{\partial y^{2}}+v_{0}^{2} \frac{\partial^{3} z_{\mathrm{b}}}{\partial x \partial y^{2}}\right] \\
& \left.+h \frac{\partial\left(h+\left(z_{\mathrm{b}} / 2\right)\right)}{\partial x}\left[-\frac{\partial^{2} u_{0}}{\partial x \partial t}-\frac{\partial^{2} v_{0}}{\partial y \partial t}-u_{0} \frac{\partial^{2} u_{0}}{\partial x^{2}}-\frac{\partial^{2}\left(u_{0} v_{0}\right)}{\partial x \partial y}-v_{0} \frac{\partial^{2} v_{0}}{\partial y^{2}}+\left(\frac{\partial u_{0}}{\partial x}+\frac{\partial v_{0}}{\partial y}\right)^{2}\right]\right] \\
& +\frac{h^{2}}{3}\left[-\frac{\partial^{3} u_{0}}{\partial x^{2} \partial t}-\frac{\partial^{3} v_{0}}{\partial x \partial y \partial t}-u_{0} \frac{\partial^{3} u_{0}}{\partial x^{3}}-\frac{\partial^{3} u_{0} v_{0}}{\partial x^{2} \partial y}-v_{0} \frac{\partial^{3} v_{0}}{\partial x \partial y^{2}}-\frac{\partial v_{0}}{\partial x}\left(\frac{\partial^{2} u_{0}}{\partial x \partial y}+\frac{\partial^{2} v_{0}}{\partial y^{2}}\right)+\left(\frac{\partial u_{0}}{\partial x}+2 \frac{\partial v_{0}}{\partial y}\right)\left(\frac{\partial^{2} v_{0}}{\partial x \partial y}+\frac{\partial^{2} u_{0}}{\partial x^{2}}\right)\right] \\
& +\left(\frac{\partial u_{0}}{\partial t}+u_{0} \frac{\partial u_{0}}{\partial x}+v_{0} \frac{\partial u_{0}}{\partial y}\right)=0
\end{aligned}
$$




\section{Numerical implementation}

To develop a valid numerical solver of 2D level I GN equations, the researchers discretised Equations 8 and 17 using second-order central finite differences (numerical solver of 2D level I GN momentum equation for non-uniform bathymetry, Equation 16 is presented in chapter 3 of the $\mathrm{PhD}$ thesis by Jalali (2016)). In the present numerical solver, two different sections were developed to deal with the continuity equation and momentum equations. Continuity equations do not have any cross-derivative terms; therefore, an explicit second-order finite difference solves the equations properly. On the other hand, momentum equations contain cross-derivative terms $\left(\partial^{2} u_{0} / \partial x \partial t\right.$ and $\left.\partial^{3} u_{0} / \partial x^{2} \partial t\right)$. Since an explicit predictor-corrector scheme is incapable of solving this kind of equation, an implicit finite difference scheme is used to solve the GN momentum equations. Herein, an implicit tridiagonal matrix inversion scheme is utilised to solve GN momentum equations (for more details see chapter 3 of the $\mathrm{PhD}$ thesis by Jalali (2016)). Nevertheless, other numerical schemes such as finite volume or finite element may be capable of solving the continuity and momentum equations simultaneously. The developed numerical solver is based on finite difference that cannot solve these equations simultaneously. The $2 \mathrm{D}$ GN continuity Equation 8 is discretised by applying second-order central difference

20.

$$
\begin{aligned}
\left.\frac{\partial h}{\partial t}\right|_{i j} ^{\mathrm{t}}= & -\left[\left(\frac{u_{0 i+1 j}^{\mathrm{t}} h_{i+1 j}^{\mathrm{t}}-u_{0 i-1 j}^{\mathrm{t}} h_{i-1 j}^{\mathrm{t}}}{2 \Delta x}\right)\right. \\
& \left.+\left(\frac{v_{0 i j+1}^{\mathrm{t}} h_{i j+1}^{\mathrm{t}}-v_{0 i j-1}^{\mathrm{t}} h_{i j-1}^{\mathrm{t}}}{2 \Delta y}\right)\right]
\end{aligned}
$$

The 2D level I GN $x$-direction momentum Equation 17 is rearranged and solved using an implicit scheme. To handle Equation 17, let

21. $\boldsymbol{F}=\frac{\partial u_{0}}{\partial t}-h\left(\frac{\partial h}{\partial x}\right) \frac{\partial}{\partial x}\left(\frac{\partial u_{0}}{\partial t}\right)-\frac{h^{2}}{3} \frac{\partial^{2}}{\partial x^{2}}\left(\frac{\partial u_{0}}{\partial t}\right)$

In second-order central differences this becomes

22

$$
\begin{aligned}
\boldsymbol{F}_{i j}^{\mathrm{t}} & =\left[h_{i j}^{\mathrm{t}}\left(\frac{h_{i+1 j}^{\mathrm{t}}-h_{i-1 j}^{\mathrm{t}}}{4 \Delta x^{2}}\right)-\frac{\left(h_{i j}^{\mathrm{t}}\right)^{2}}{3 \Delta x^{2}}\right] \hat{u}_{0_{i-1 j}}^{\mathrm{t}} \\
+ & {\left[1+\frac{2\left(h_{i j}^{\mathrm{t}}\right)^{2}}{3 \Delta x^{2}}\right] \hat{u}_{0_{i j}}^{\mathrm{t}}+\left[-h_{i j}^{\mathrm{t}}\left(\frac{h_{i+1 j}^{\mathrm{t}}-h_{i-1 j}^{\mathrm{t}}}{4 \Delta x^{2}}\right)-\frac{\left(h_{i j}^{\mathrm{t}}\right)^{2}}{3 \Delta x^{2}}\right] \hat{u}_{0_{i+1 j}}^{\mathrm{t}} }
\end{aligned}
$$

where $\hat{u}_{0_{i j}}^{\mathrm{t}}=\partial u_{0} /\left.\partial t\right|_{i j} ^{\mathrm{t}}$. The discretised equation is rewritten as

23. $\boldsymbol{F}_{i j}^{\mathrm{t}}=a_{i j}^{\mathrm{t}} \hat{u}_{0_{i-1 j}}^{\mathrm{t}}+b_{i j}^{\mathrm{t}} \hat{u}_{0_{i j}}^{\mathrm{t}}+c_{i j}^{\mathrm{t}} \hat{u}_{0_{i+1 j}}^{\mathrm{t}}$ where $a_{i j}^{\mathrm{t}}, b_{i j}^{\mathrm{t}}$ and $c_{i j}^{\mathrm{t}}$ are the tridiagonal matrix coefficients for $x$-direction. Here, $i$ refers to $x$-direction; $j$ is $y$-direction and $t$ is time. $\boldsymbol{F}$ is also equal to the remaining spatial derivative terms in Equation 17. These terms are also discretised in Equation 17 by using second-order central differences, giving

24.

$$
\begin{aligned}
\boldsymbol{F}_{i j}^{\mathrm{t}} & =-\left[\left.u_{0 i j}^{\mathrm{t}} \frac{\partial u_{0}}{\partial x}\right|_{i j} ^{\mathrm{t}}+\left.v_{0 i j}^{\mathrm{t}} \frac{\partial u_{0}}{\partial y}\right|_{i j} ^{\mathrm{t}}+\left.\boldsymbol{g} \frac{\partial \eta}{\partial x}\right|_{i j} ^{\mathrm{t}}\right] \\
& +\left.h_{i j}^{\mathrm{t}} \frac{\partial h}{\partial x}\right|_{i j} ^{\mathrm{t}}\left[\left.u_{0 i j}^{\mathrm{t}} \frac{\partial^{2} u_{0}}{\partial x^{2}}\right|_{i j} ^{\mathrm{t}}+\left.\frac{\partial^{2}\left(u_{0} v_{0}\right)}{\partial x \partial y}\right|_{i j} ^{\mathrm{t}}+\left.v_{0 i j}^{\mathrm{t}} \frac{\partial^{2} v_{0}}{\partial y^{2}}\right|_{i j} ^{\mathrm{t}}\right. \\
& \left.-\left(\left.\frac{\partial u_{0}}{\partial x}\right|_{i j} ^{\mathrm{t}}+\left.\frac{\partial v_{0}}{\partial y}\right|_{i j} ^{\mathrm{t}}\right)^{2}\right]+\frac{h_{i j}^{\mathrm{t}}}{3}\left[\left.u_{0 i j}^{\mathrm{t}} \frac{\partial^{3} u_{0}}{\partial x^{3}}\right|_{i j} ^{\mathrm{t}}+\left.\frac{\partial^{3} u_{0} v_{0}}{\partial x^{2} \partial y}\right|_{i j} ^{\mathrm{t}}\right. \\
& +\left.\frac{\partial v_{0}}{\partial x}\right|_{i j} ^{\mathrm{t}}\left(\left.\frac{\partial^{2} u_{0}}{\partial x \partial y}\right|_{i j} ^{\mathrm{t}}+\left.\frac{\partial^{2} v_{0}}{\partial y^{2}}\right|_{i j} ^{\mathrm{t}}\right)+\left.v_{0 i j}^{\mathrm{t}} \frac{\partial^{3} v_{0}}{\partial x \partial y^{2}}\right|_{i j} ^{\mathrm{t}} \\
& \left.-\left(\left.\frac{\partial u_{0}}{\partial x}\right|_{i j} ^{\mathrm{t}}+\left.2 \frac{\partial v_{0}}{\partial y}\right|_{i j} ^{\mathrm{t}}\right)\left(\left.\frac{\partial^{2} v_{0}}{\partial x \partial y}\right|_{i j} ^{\mathrm{t}}+\left.\frac{\partial^{2} u_{0}}{\partial x^{2}}\right|_{i j} ^{\mathrm{t}}\right)\right]^{\mathrm{t}} \\
& +\left.\left.h_{i j}^{\mathrm{t}} \frac{\partial h}{\partial x}\right|_{i j} ^{\mathrm{t}} \frac{\partial}{\partial y}\left(\frac{\partial v_{0}}{\partial t}\right)\right|_{i j} ^{\mathrm{t}}+\left.\frac{h_{i j}^{\mathrm{t} 2}}{3} \frac{\partial^{2}}{\partial x \partial y}\left(\frac{\partial v_{0}}{\partial t}\right)\right|_{i j} ^{\mathrm{t}}
\end{aligned}
$$

The above sets of discretised equations form the tridiagonal matrix system. The unknown values $\hat{u}_{0_{i j}}^{\mathrm{t}}$ are obtained for $j=2, \ldots, j_{\max }-1$ and $i=1, \ldots, i_{\max }$ using the Thomas algorithm (Press et al., 2007). It should be mentioned that $i_{\max }$ and $j_{\max }$ refer to the maximum number of grid points in $x$ - and $y$-directions. A similar numerical approach was applied to $2 \mathrm{D}$ level I GN $y$-momentum equation (for a detailed development of GN numerical solver refer to chapter 3 of the $\mathrm{PhD}$ thesis by Jalali (2016)). Iteration is then used to centre correctly (in space and time) the cross-derivative terms that appear in both the $x$ - and $y$-direction momentum equations. RungeKutta fourth-order time integration is used to update the total depth and horizontal velocity components at each time step.

\section{Boundary conditions}

To solve the $2 \mathrm{D}$ GN equations, it is necessary to impose flexible and compatible boundary conditions. For instance, solid wall boundaries are located at the ends of the domain when simulating sloshing of waves in a tank. The surface elevation at the boundary obtained by cubic Lagrange interpolation of interior values is assigned according to Haniffah (2013). The velocity is set to zero at solid wall boundaries. Additional ghost grid points are located outside the boundaries, with antisymmetry imposed for horizontal velocity $(u)$ on $y$-direction and symmetry on $x$-direction. 
The velocity boundary conditions on $x$-direction for numerical solver of 2D GN equations are

$$
\begin{aligned}
& \left.\frac{\partial u_{0}}{\partial t}\right|_{1, j} ^{\mathrm{t}}=0,\left.\quad \frac{\partial u_{0}}{\partial t}\right|_{0, j} ^{\mathrm{t}}=-\left.\frac{\partial u_{0}}{\partial t}\right|_{2, j} ^{\mathrm{t}},\left.\quad \frac{\partial u_{0}}{\partial t}\right|_{-1, j} ^{\mathrm{t}}=-\left.\frac{\partial u_{0}}{\partial t}\right|_{3, j} ^{\mathrm{t}} \\
& \left.\frac{\partial u_{0}}{\partial t}\right|_{i_{\max }, j} ^{\mathrm{t}}=0,\left.\quad \frac{\partial u_{0}}{\partial t}\right|_{i_{\max }+1, j} ^{\mathrm{t}}=-\left.\frac{\partial u_{0}}{\partial t}\right|_{i_{\max }-1, j} ^{\mathrm{t}} \\
& \left.\frac{\partial u_{0}}{\partial t}\right|_{i_{\max }+2, j} ^{\mathrm{t}}=-\left.\frac{\partial u_{0}}{\partial t}\right|_{i_{\max }-2, j} ^{\mathrm{t}},\left.\quad \frac{\partial u_{0}}{\partial t}\right|_{i, 1} ^{\mathrm{t}}=0 \\
& \left.\frac{\partial u_{0}}{\partial t}\right|_{i, 0} ^{\mathrm{t}}=\left.\frac{\partial u_{0}}{\partial t}\right|_{i, 2} ^{\mathrm{t}},\left.\quad \frac{\partial u_{0}}{\partial t}\right|_{i,-1} ^{\mathrm{t}}=\left.\frac{\partial u_{0}}{\partial t}\right|_{i, 3} ^{\mathrm{t}},\left.\quad \frac{\partial u_{0}}{\partial t}\right|_{i, j_{\max }} ^{\mathrm{t}}=0 \\
& \left.\frac{\partial u_{0}}{\partial t}\right|_{i, j_{\max }+1} ^{\mathrm{t}}=\left.\frac{\partial u_{0}}{\partial t}\right|_{i, j_{\max }-1} ^{\mathrm{t}},\left.\quad \frac{\partial u_{0}}{\partial t}\right|_{i, j_{\max }+2} ^{\mathrm{t}}=\left.\frac{\partial u_{0}}{\partial t}\right|_{i, j_{\max }-2} ^{\mathrm{t}}
\end{aligned}
$$

Here, index $1, i_{\max }$ and $j_{\max }$ present the location of the wall; index $2,3, i_{\max }-1, i_{\max }-2, j_{\max }-1$ and $j_{\max }-2$ indicate the grid points located inside of the computational boundary (before the wall); and index $-1,0, i_{\max }+1, i_{\max }+2, j_{\max }+1$ and $j_{\max }+2$ show ghost points located outside of the computational boundary (after the wall). The velocity boundary conditions in $y$-direction are not included here for brevity (for more details see Jalali (2016)). The symmetry boundaries imposed for surface elevation are

$$
\begin{gathered}
\left.\frac{\partial h}{\partial t}\right|_{0, j} ^{\mathrm{t}}=\left.\frac{\partial h}{\partial t}\right|_{2, j} ^{\mathrm{t}},\left.\quad \frac{\partial h}{\partial t}\right|_{i_{\max }+1, j} ^{\mathrm{t}}=\left.\frac{\partial h}{\partial t}\right|_{i_{\max }-1, j} ^{\mathrm{t}} \\
\left.\frac{\partial h}{\partial t}\right|_{1, j} ^{\mathrm{t}}=\left.4 \frac{\partial h}{\partial t}\right|_{2, j} ^{\mathrm{t}}-\left.6 \frac{\partial h}{\partial t}\right|_{3, j} ^{\mathrm{t}}+\left.4 \frac{\partial h}{\partial t}\right|_{4, j} ^{\mathrm{t}}-\left.\frac{\partial h}{\partial t}\right|_{5, j} ^{\mathrm{t}} \\
\left.\frac{\partial h}{\partial t}\right|_{i_{\max }, j} ^{\mathrm{t}}=\left.4 \frac{\partial h}{\partial t}\right|_{i_{\max }-1, j} ^{\mathrm{t}}-\left.6 \frac{\partial h}{\partial t}\right|_{i_{\max }-2, j} ^{\mathrm{t}} \\
\quad+\left.4 \frac{\partial h}{\partial t}\right|_{i_{\max }-3, j} ^{\mathrm{t}}-\left.\left.\frac{\partial h}{\partial t}\right|_{i_{\max }-4, j} ^{\mathrm{t}}\right|_{i, 0} ^{\mathrm{t}}= \\
\left.\frac{\partial h}{\partial t}\right|_{i, 2} ^{\mathrm{t}},\left.\quad \frac{\partial h}{\partial t}\right|_{i, j_{\max }+1} ^{\mathrm{t}}=\left.\frac{\partial h}{\partial t}\right|_{i, j_{\max }-1} ^{\mathrm{t}}=\left.4 \frac{\partial h}{\partial t}\right|_{i, 2} ^{\mathrm{t}}-\left.6 \frac{\partial h}{\partial t}\right|_{i, 3} ^{\mathrm{t}}+\left.4 \frac{\partial h}{\partial t}\right|_{i, 4} ^{\mathrm{t}}-\left.\frac{\partial h}{\partial t}\right|_{i, 5} ^{\mathrm{t}} \\
\left.\frac{\partial h}{\partial t}\right|_{i, j_{\max }} ^{\mathrm{t}}=\left.4 \frac{\partial h}{\partial t}\right|_{i, j_{\max }-1} ^{\mathrm{t}}-\left.6 \frac{\partial h}{\partial t}\right|_{i, j_{\max }-2} ^{\mathrm{t}} \\
+\left.4 \frac{\partial h}{\partial t}\right|_{i, j_{\max }-3} ^{\mathrm{t}}-\left.\frac{\partial h}{\partial t}\right|_{i, j_{\max }-4} ^{\mathrm{t}}
\end{gathered}
$$

In 1D level I GN numerical solver, Equations 18 and 19 are the governing equations. The 1D GN continuity Equation 19 is discretised by applying the second-order central difference

$$
\text { 25. }\left.\frac{\partial h}{\partial t}\right|_{i} ^{\mathrm{t}}=-\left(\frac{u_{0 i+1}^{\mathrm{t}} h_{i+1}^{\mathrm{t}}-u_{0 i-1}^{\mathrm{t}} h_{i-1}^{\mathrm{t}}}{2 \Delta x}\right)
$$

In Equation 18, cross-derivative terms of $x$ and $t$ are rearranged and solved by using an implicit scheme, similar to the procedure followed for Equation 21. The discretised equation is

26. $\quad \boldsymbol{F}_{i}^{\mathrm{t}}=a_{i}^{\mathrm{t}} \hat{u}_{0_{i-1}}^{\mathrm{t}}+b_{i}^{\mathrm{t}} \hat{u}_{0_{i}}^{\mathrm{t}}+c_{i}^{\mathrm{t}} \hat{u}_{0_{i+1}}^{\mathrm{t}}$

$\boldsymbol{F}$ is also equal to the remaining spatial derivative terms in Equation 18. Using second-order central differences discretisise the terms in Equation 18 and gives

27.

$$
\begin{aligned}
\boldsymbol{F}_{i}^{\mathrm{t}}= & \left.-\left.u_{0 i}^{\mathrm{t}} \frac{\partial u_{0}}{\partial x}\right|_{i} ^{\mathrm{t}}-\left.\boldsymbol{g} \frac{\partial \eta}{\partial x}\right|_{i} ^{\mathrm{t}}+\left.\left.\left.h_{i}^{\mathrm{t}} \frac{\partial h}{\partial x}\right|_{i} ^{\mathrm{t}}\right|_{0 i} ^{\mathrm{t}} \frac{\partial^{2} u_{0}}{\partial x^{2}}\right|_{i} ^{\mathrm{t}}-\left.\left(\frac{\partial u_{0}}{\partial x}\right)^{2}\right|_{i} ^{\mathrm{t}}\right]^{\mathrm{t}}{ }^{\mathrm{t} 2}\left[\left.u_{0} i^{\mathrm{t}} \frac{\partial^{3} u_{0}}{\partial x^{3}}\right|_{i} ^{\mathrm{t}}-\left.\left.\frac{\partial u_{0}}{\partial x}\right|_{i} ^{\mathrm{t}} \frac{\partial^{2} u_{0}}{\partial x^{2}}\right|_{i} ^{\mathrm{t}}\right]
\end{aligned}
$$

The velocity boundary conditions for solver of $1 \mathrm{D}$ GN equation are

$$
\begin{aligned}
& \left.\frac{\partial u_{0}}{\partial t}\right|_{1} ^{\mathrm{t}}=0,\left.\quad \frac{\partial u_{0}}{\partial t}\right|_{0} ^{\mathrm{t}}=-\left.\frac{\partial u_{0}}{\partial t}\right|_{2} ^{\mathrm{t}},\left.\quad \frac{\partial u_{0}}{\partial t}\right|_{-1} ^{\mathrm{t}}=-\left.\frac{\partial u_{0}}{\partial t}\right|_{3} ^{\mathrm{t}} \\
& \left.\frac{\partial u_{0}}{\partial t}\right|_{i_{\max }} ^{\mathrm{t}}=0,\left.\quad \frac{\partial u_{0}}{\partial t}\right|_{i_{\max }+1} ^{\mathrm{t}}=-\left.\frac{\partial u_{0}}{\partial t}\right|_{i_{\max }-1} ^{\mathrm{t}} \\
& \left.\frac{\partial u_{0}}{\partial t}\right|_{i_{\max }+2} ^{\mathrm{t}}=-\left.\frac{\partial u_{0}}{\partial t}\right|_{i_{\max }-2} ^{\mathrm{t}}
\end{aligned}
$$

In 1D GN numerical solver, the symmetry boundaries imposed for surface elevation are

$$
\begin{aligned}
& \left.\frac{\partial h}{\partial t}\right|_{0} ^{\mathrm{t}}=\left.\frac{\partial h}{\partial t}\right|_{2} ^{\mathrm{t}},\left.\quad \frac{\partial h}{\partial t}\right|_{i_{\max }+1} ^{\mathrm{t}}=\left.\frac{\partial h}{\partial t}\right|_{i_{\max }-1} ^{\mathrm{t}} \\
& \left.\frac{\partial h}{\partial t}\right|_{1} ^{\mathrm{t}}=\left.4 \frac{\partial h}{\partial t}\right|_{2} ^{\mathrm{t}}-\left.6 \frac{\partial h}{\partial t}\right|_{3} ^{\mathrm{t}}+\left.4 \frac{\partial h}{\partial t}\right|_{4} ^{\mathrm{t}}-\left.\frac{\partial h}{\partial t}\right|_{5} ^{\mathrm{t}} \\
& \left.\frac{\partial h}{\partial t}\right|_{i_{\max }} ^{\mathrm{t}}=\left.4 \frac{\partial h}{\partial t}\right|_{i_{\max }-1} ^{\mathrm{t}}-\left.6 \frac{\partial h}{\partial t}\right|_{i_{\max }-2} ^{\mathrm{t}}+\left.4 \frac{\partial h}{\partial t}\right|_{i_{\max }-3} ^{\mathrm{t}}-\left.\frac{\partial h}{\partial t}\right|_{i_{\max }-4} ^{\mathrm{t}}
\end{aligned}
$$

For more details, see chapter 3 of the $\mathrm{PhD}$ thesis by Jalali (2016) and chapter 4 of the $\mathrm{PhD}$ thesis by Haniffah (2013). 


\section{Numerical procedure}

The GN program comprises four main subroutines: input, calculation, update and output. For each test case, the following initial values are put into the program: bed elevation, initial water depth, amplitude, length and width of the study basin, number of grid points, time step and duration of simulation time. The initial conditions supply for the bed elevation above fixed horizontal datum, local depth and local horizontal velocity components throughout the tank. Solving the discretised continuity equation provides new water depth values throughout the grid. Then, the discretised momentum equations are solved. Iteration is used to solve the cross-derivative velocity terms in the momentum equation. Next, boundary conditions are invoked. The values of $u, v$ and $h$ are updated after each time step. The calculation process is repeated until the simulation is complete. The two benchmark tests comprise: sloshing in a square tank and free surface sloshing of an initial Gaussian hump in a square basin. In sloshing in a square tank to test for grid independence, the time history of wave elevation at the corner of the tank (in positive $x$-direction) was obtained on grids of increasingly fine resolution ( $\Delta x=25 \mathrm{~m}$ (coarse grids), $\Delta x=10 \mathrm{~m}$ (medium grids) and $\Delta x=1 \mathrm{~m}$ (fine grids)) and a fixed time step $\Delta t=1 \mathrm{~s}$. The results demonstrated that $\Delta x=1 \mathrm{~m}$ was sufficient to achieve a converged solution. Three time steps are chosen $(\Delta t=0.25,1.0$ and $2.0 \mathrm{~s})$ on the converged grid with $\Delta x=1 \mathrm{~m}$. There was close agreement between the results; therefore, $\Delta t=1 \mathrm{~s}$ was selected as a fixed time step in the simulation of sloshing in the tank (see chapter 4 of the $\mathrm{PhD}$ thesis by Jalali (2016)). To determine the number of grid points required to produce an accurate simulation of free surface sloshing of an initial Gaussian hump, grid convergence test was performed. To this end, 3D visualisations and contour maps of the free surface elevation patterns in the basin were obtained on increasing grid size with $\Delta x=\Delta y=0.15 \mathrm{~m}$ (coarse grids), $\Delta x=\Delta y=0.0375 \mathrm{~m}$ (medium grids) and $\Delta x=\Delta y=7.5 \times 10^{-3} \mathrm{~m}$ (fine grids). The medium grid size $\Delta x=\Delta y=0.0375 \mathrm{~m}$ is sufficient for convergence. Therefore, $\Delta x=\Delta y=0.0375 \mathrm{~m}$ were chosen for numerical simulations. The numerical predictions of free surface sloshing of an initial Gaussian hump for different time steps $(\Delta t=0.05,0 \cdot 1$ and $0.2 \mathrm{~s})$ have shown that $\Delta t=0.05 \mathrm{~s}$ was sufficient for producing an accurate simulation (see chapter 5 of the PhD thesis by Jalali (2016)).

\section{Model verification against analytical solution}

\subsection{Sloshing in a tank}

First, the benchmark test of sinusoidal free surface sloshing in a square tank is considered. The wavelength, $L$, is $1000 \mathrm{~m}$ and the still water depth, $h_{0}$, is $5 \mathrm{~m}$. Sloshing motions may even occur by using a very small number of amplitude disturbance. For the present test case the amplitudes are $a=0.005$ and $0.05 \mathrm{~m}$. These small numbers of wave amplitude are applied in order to create minimum non-linear behaviour by the sloshing wave. The first-order analytical solution for the depth profile evolution in space and time of a standing wave in a tank (e.g. Dean and Dalrymple, 2004) is

28. $\zeta_{\mathrm{c}}=h_{\mathrm{c}}-h_{0}=a \cos (k x) \cos (\omega t+\phi)$

Here, $\zeta_{\mathrm{c}}$ refers to the crest-induced free surface elevation time series, $a$ is the amplitude of the standing wave, $k$ is the wave number, $\omega$ is the angular frequency of the wave, $x$ is the distance along the tank, $t$ is time and $\phi$ is the phase. Wave angular frequency $\omega$ is obtained by means of dispersion relation

29. $\omega=\sqrt{g k \tanh k h_{0}}$

In this case: $\omega=0.044 \mathrm{rad} / \mathrm{s}, T($ period $)=2 \pi / \omega=142.8 \mathrm{~s}$ and $f=($ frequency $)=0.007 \mathrm{~Hz}$.

Figures 1 and 2 are obtained by applying the numerical solver of $1 \mathrm{D}$ GN equations. This program was specifically developed

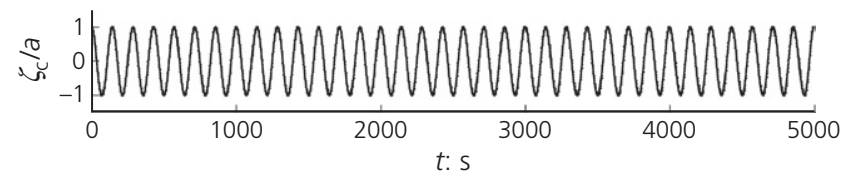

(a)

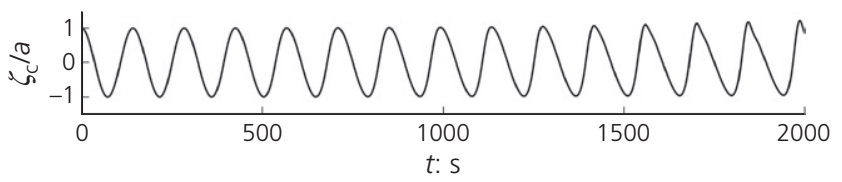

(b)

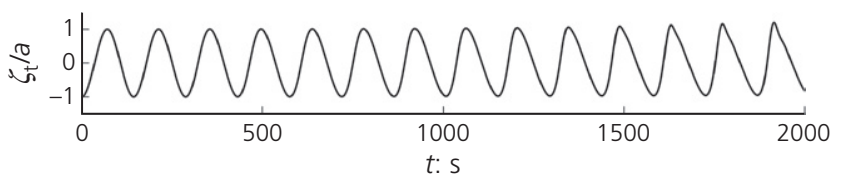

(c)

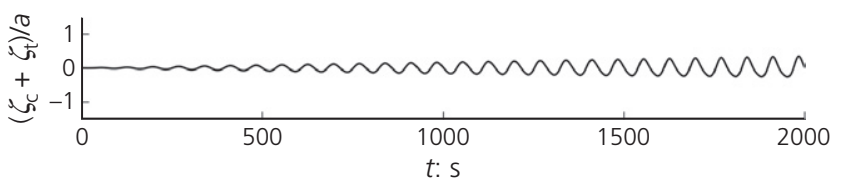

(d)

Figure 1. Sinusoidal free surface sloshing at the corner of a square tank: (a) analytical (solid line) and GN-predicted (cross symbols) crest-induced free surface time histories for $a=0.005 \mathrm{~m}$; (b) GN-predicted crest-induced free surface time history for $a=0.05 \mathrm{~m}$; (c) GN-predicted trough-induced free surface time history for $a=0.05 \mathrm{~m}$; and (d) GN-predicted even harmonics time history for $a=0.05 \mathrm{~m}$ 


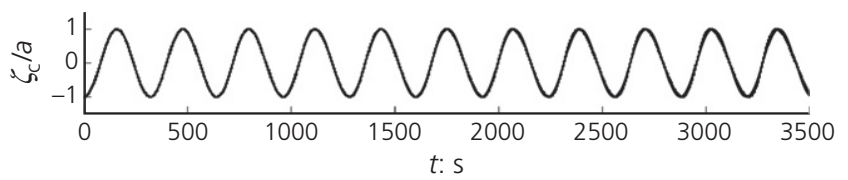

(a)

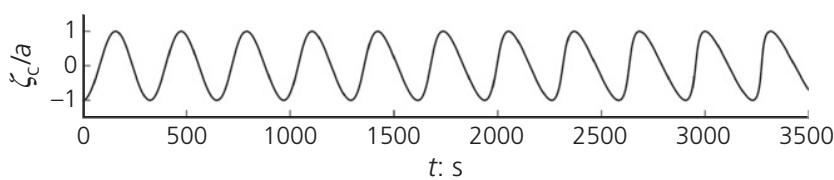

(b)

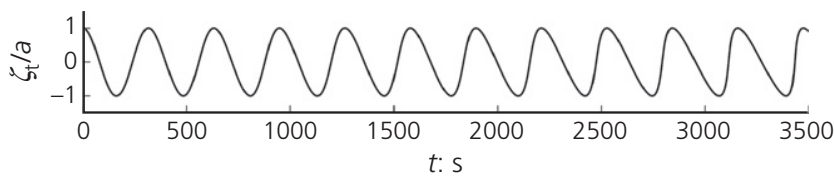

(c)

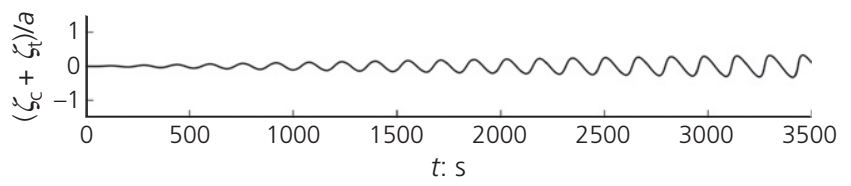

(d)

Figure 2. Sinusoidal free surface sloshing at the centre of a square tank: (a) analytical (solid line) and GN-predicted (cross symbols) crest-induced free surface time histories for $a=0.005 \mathrm{~m}$; (b) GN-predicted crest-induced free surface time history for $a=0.015 \mathrm{~m}$; (c) GN-predicted trough-induced free surface time history for $a=0.015 \mathrm{~m}$; and (d) GN-predicted even harmonics time history for $a=0.015 \mathrm{~m}$

to deal with sloshing in the tank, so it is necessary to measure free surface elevation of time history at two different locations: $(a)$ in the corner of the tank and $(b)$ at the centre of the tank. Figure 1(a) presents crest-induced water level time histories in the corner of the tank (in positive $x$-direction) for a sloshing wave of a small-amplitude disturbance $a=0.005 \mathrm{~m}$ with phase $\phi=0$. Here, $\zeta_{\mathrm{c}} / a=1$ at time $t=0 \mathrm{~s}$ for free surface elevation of time history in the corner of tank. Excellent agreement is obtained between the first-order analytical solution and the numerical prediction in which cross symbols (numerical prediction) essentially overlay the solid line (analytical solution). The standing wave behaviour is periodic and of constant amplitude. This case verifies that the numerical scheme gives a correct representation of the underlying mathematical description, provided the waves are nearly linear. Figure 1(b) depicts the numerical prediction of the crest-induced free surface elevation time history in the corner of the tank for $a=0.05 \mathrm{~m}$. The free surface elevation time history displayed in Figure 1(b) is shorter than that in Figure 1(a) because non-linear effects eventually cause shock-like steepening of the wave profiles (becoming visible at about $t=2000 \mathrm{~s}$ ) in the larger amplitude case leading to the numerical model becoming unstable. A shock-capturing scheme would be needed to overcome this problem, and is recommended for future implementation. Figure 1(c) presents the numerically predicted trough-induced free surface elevation time history at the corner of the tank in which $\zeta_{\mathrm{t}}=-a \cos (k x) \cos (\omega t)$. Qualitatively, the results are almost the same as for the crest-induced case (i.e. Figure1(b)). Non-linearity can be presented by even harmonics $\left(\zeta_{\mathrm{c}}+\zeta_{\mathrm{t}} / 2 a\right)$. To separate even harmonics, harmonics are treated as orthogonal functions. Figure 1(d) shows the numerically predicted free surface elevation time history of the even harmonic components (obtained by taking the average of results obtained for $\phi=0$ and $\pi$ ) for the amplitude $a=0.05 \mathrm{~m}$. Here, the amplitude of the second-order harmonics grows monotonically with time until the point at which numerical instability occurs. It is worth noting that the (linear) analytical solution is not able to show the non-linear behaviour of even harmonics. The developed numerical model is also applied to simulate other possible predictions for a sloshing wave in the centre of the tank. In this case, the wavelength is $1000 \mathrm{~m}$ and the still water depth $h_{0}$ is $1 \mathrm{~m}$. Two values of wave amplitude, $a=0.005$ and $0.015 \mathrm{~m}$, are selected. In this case: $\omega=0.0197 \mathrm{rad} / \mathrm{s}, \quad T=319.28 \mathrm{~s}$ and $f=0.003 \mathrm{~Hz}$.

Figure 2(a) shows satisfactory agreement between the numerical prediction (cross symbols) and the analytical solution (solid line) of the crest-induced free surface elevation time histories at the centre of the tank by applying $a=0.005 \mathrm{~m}$. Here, $\zeta_{\mathrm{c}} / a=-1$ at time $t=0 \mathrm{~s}$ for the crest-induced free surface elevation of time history at the centre of the tank. Figure 2(b) depicts the numerically predicted crest-induced free surface elevation time history for $a=0.015 \mathrm{~m}$. It is clear that the numerical solver is unable to simulate the long-term sloshing behaviour of the wave, with high-order even harmonic oscillations appearing after $t=1735 \mathrm{~s}$. The non-linear effects eventually caused shock-like steepening of the wave profiles. Therefore, a shock-capturing scheme is required to overcome this problem. Figure 2(c) shows the numerically predicted free surface elevation time history of the trough-induced sloshing at the centre of the tank for $a=0.015 \mathrm{~m}$. The results are qualitatively almost the same as for the crest-induced case (i.e. Figure 2(b)). Figure 2(d) shows the numerically predicted free surface elevation time history of the even harmonic components (obtained by $\zeta_{\mathrm{c}}+\zeta_{\mathrm{t}} / 2 a$ ) for $a=0.015 \mathrm{~m}$. The effect of non-linearity increases as the initial slosh amplitude increases, as would be expected (for more details see chapter 4 of the $\mathrm{PhD}$ thesis by Jalali (2016)).

\subsection{Free surface sloshing of an initial Gaussian hump in a closed square, flat-bottomed basin}

The numerical solver of the 2D GN equations is now verified for non-linear free surface sloshing motions arising 
Table 1. Initial Gaussian free surface hump in a square basin: elevation of free surface perturbation $\zeta_{\mathrm{c}} / a$ at the centre of basin at time $t=0 \mathrm{~s}$ for different numbers of wave components $(p, q)$ and grid size $(\Delta x, \Delta y)$

\begin{tabular}{lccc|} 
& \multicolumn{3}{c}{$\Delta \boldsymbol{x}=\Delta \boldsymbol{y}$} \\
$\boldsymbol{p}=\boldsymbol{q}$ & $0.3 \mathrm{~m}$ & $0.075 \mathrm{~m}$ & $0.015 \mathrm{~m}$ \\
\hline 51 & 0.022 & 1 & 1 \\
101 & 0.022 & 0.355 & 1 \\
\hline
\end{tabular}

from an initial Gaussian hump free surface profile in a closed basin. The well-established analytical solution (Lamb, 1916; Wei and Kirby, 1995; Yao, 2007) of the linearised SWEs for the evolution of an initial hump in a closed square basin is

30. $\zeta(x, y, t)=\sum_{p=0}^{r=\infty} \sum_{q=0}^{r=\infty} \tilde{\zeta}_{p q} \mathrm{e}^{-i \omega t} \times \cos \left(\frac{p \pi}{L_{x}} x\right) \cos \left(\frac{q \pi}{L_{y}} y\right)$

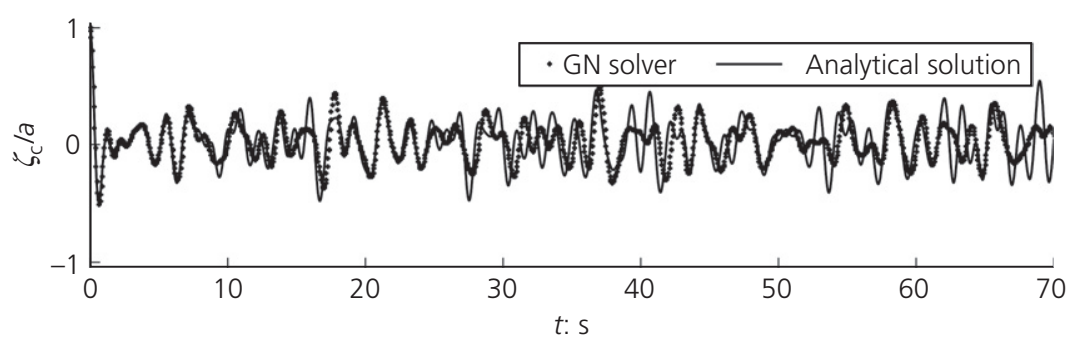

Figure 3. Analytical and predicted free surface elevation time histories at the centre of a basin for sloshing of an initial Gaussian hump in a square, flat-bottomed basin applying $a=0.045 \mathrm{~m}$ and $b=2 \mathrm{~m}^{-2}$

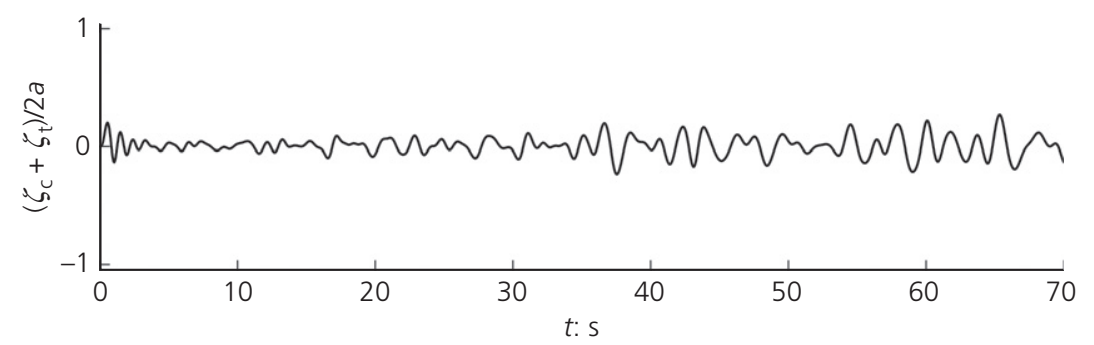

Figure 4. Free surface elevation time history of even harmonics component of an initial Gaussian hump for amplitude $a=h_{0} / 2=0.225 \mathrm{~m}$ at the centre of a square, flat-bottomed basin

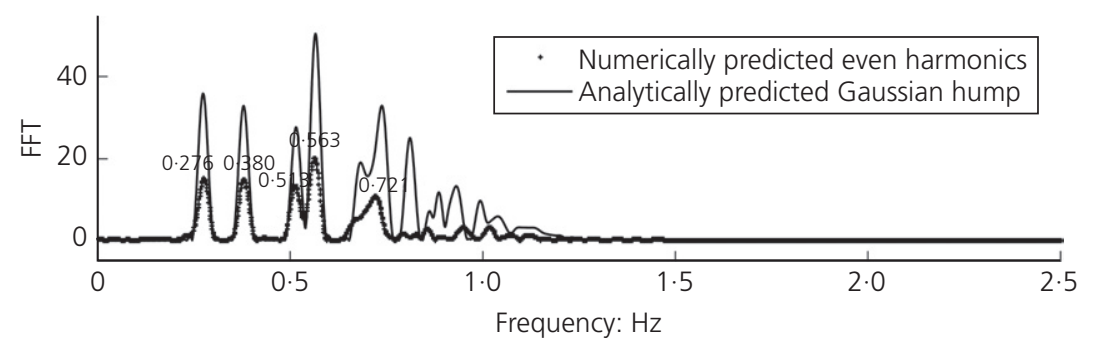

Figure 5. Comparison between analytically predicted fast Fourier transform (FFT) spectrum for the free surface elevation time history of the initial Gaussian hump with the numerically predicted FFT spectrum for the free surface elevation time history of even harmonics component 
Engineering and Computational Mechanics

Volume 170 Issue EM2
One-dimensional and two-dimensional Green-Naghdi equations for sloshing

in shallow basins

Jalali and Borthwick
Table 2. Analytical fundamental sloshing frequencies in a square basin (all values in $\mathrm{Hz}$ )

\begin{tabular}{cccccc} 
& \multicolumn{5}{c}{$\boldsymbol{r}$} \\
$\boldsymbol{n}$ & 1 & 2 & 3 & 4 & 5 \\
\hline 0 & 0.140 & 0.280 & 0.420 & 0.560 & 0.700 \\
1 & 0.198 & 0.313 & 0.442 & 0.577 & 0.714 \\
2 & 0.313 & 0.396 & 0.505 & 0.626 & 0.754 \\
3 & 0.442 & 0.505 & 0.594 & 0.700 & 0.816 \\
4 & 0.577 & 0.626 & 0.700 & 0.792 & 0.896 \\
5 & 0.714 & 0.754 & 0.816 & 0.896 & 0.990
\end{tabular}

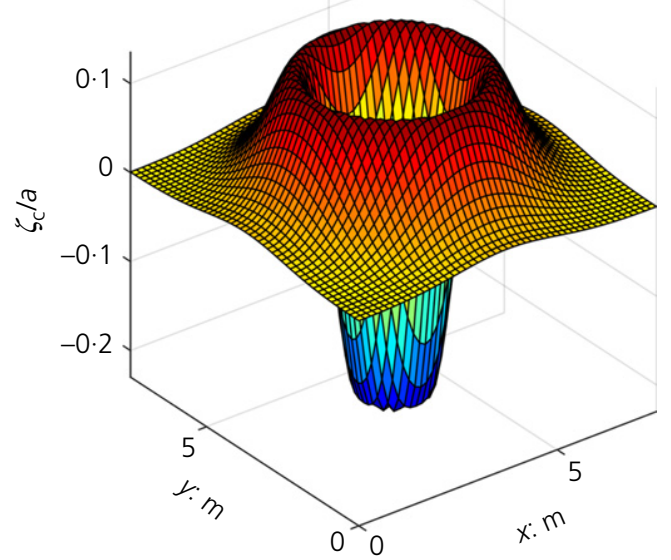

(a)

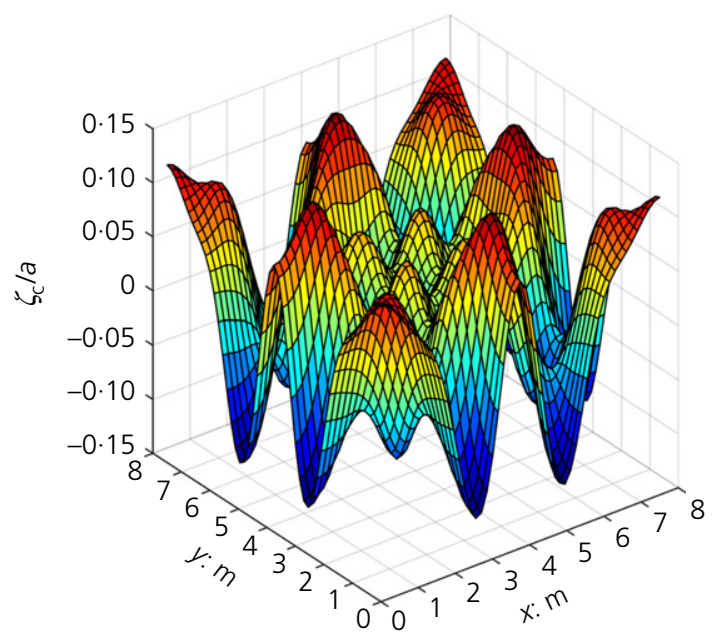

(c)
Here, $\zeta$ is the free surface elevation above still water level, where

31.

$$
\begin{aligned}
\tilde{\zeta}_{p q}= & \frac{4}{\left(1+\sigma_{p 0}\right)\left(1+\sigma_{q 0}\right) L_{x} L_{y}} \\
& \times \int_{-L_{y}}^{L_{y}} \int_{-L_{x}}^{L_{x}} \zeta_{0}(x, y) \cos \left(\frac{p \pi}{L_{x}} x\right) \cos \left(\frac{q \pi}{L_{y}} y\right) \mathrm{d} x \mathrm{~d} y
\end{aligned}
$$

In Equation 30, $i=\sqrt{-1} ; \quad L_{x}$ and $L_{y}$ are the length and width of the basin; $\omega$ is angular frequency; $p$ and $q$ are the number of wave components; $\sigma_{p q}$ is the Kronecker delta

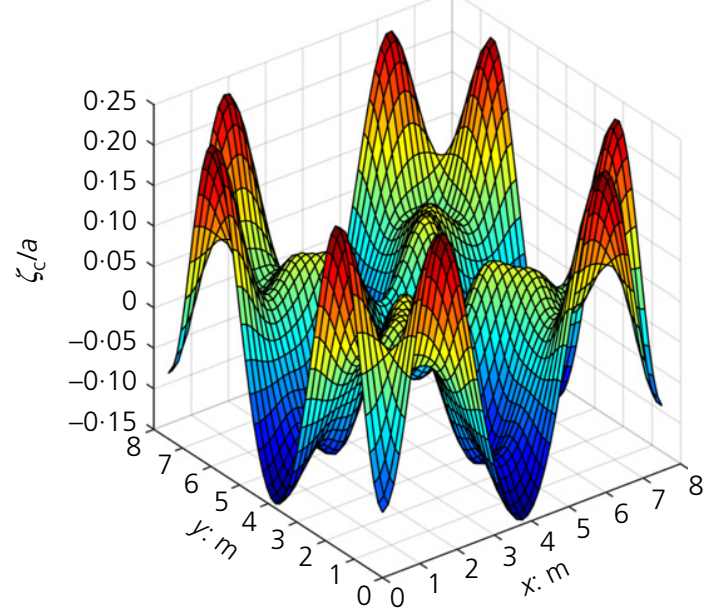

(b)

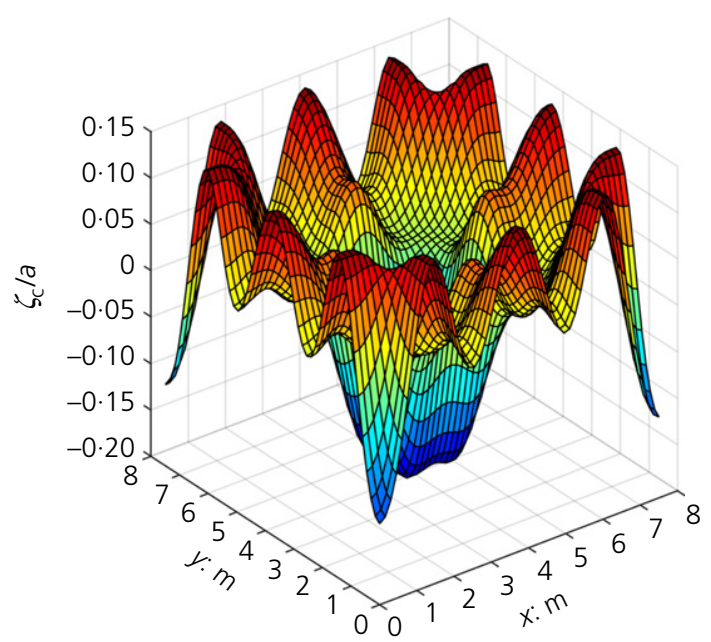

(d)

Figure 6. Predicted 3D visualisations of the sloshing of an initial Gaussian hump in a square, flat-bottomed basin: (a) $t=1 \mathrm{~s}$; (b) $t=5 \mathrm{~s}$; (c) $t=10 \mathrm{~s}$; and (d) $t=20 \mathrm{~s}$ 
function and

32. $\zeta_{0}(x, y)=a \exp \left\{-b\left[\left(x-\frac{L_{x}}{2}\right)^{2}+\left(y-\frac{L_{y}}{2}\right)^{2}\right]\right\}$

where $a$ is the wave amplitude and $b$ is the spreading parameter.

Consider a basin of $7.5 \mathrm{~m}$ length and $7.5 \mathrm{~m}$ width. The constant water depth is $h_{0}=0.45 \mathrm{~m}$. The initial amplitude of the hump $a$ is $0.045 \mathrm{~m}$ and the spreading parameter $b=2 \mathrm{~m}^{-2}$. To obtain an accurate estimate of the analytical solution, different numbers of wave components $(p$ and $q)$ and grid size $(\Delta x$ and $\Delta y$ ) are selected to solve the double Fourier series in Equation 30. Table 1 presents the initial elevation of analytically predicted free surface perturbation $\zeta_{\mathrm{c}} / a$ at the centre of the basin at time $t=0 \mathrm{~s}$ for different numbers of wave components $(p, q)$ and grid sizes $(\Delta x, \Delta y)$. This table shows that using $p=q=51$ and $\Delta x=\Delta y=0.075 \mathrm{~m}$ is sufficient to obtain a converged analytical solution. In the present GN numerical solver, four iterations on the medium grid size, $\Delta x=\Delta y=0.0375 \mathrm{~m}$, are sufficient for the numerical predictions to be in satisfactory agreement with the analytical results. A time step of $\Delta t=0.05 \mathrm{~s}$ is found to be sufficient to achieve accurate and stable results. Figure 3 compares the numerical free surface elevation time history with the analytical solution at the centre of the basin for a total simulation time of $70 \mathrm{~s}$ after release of

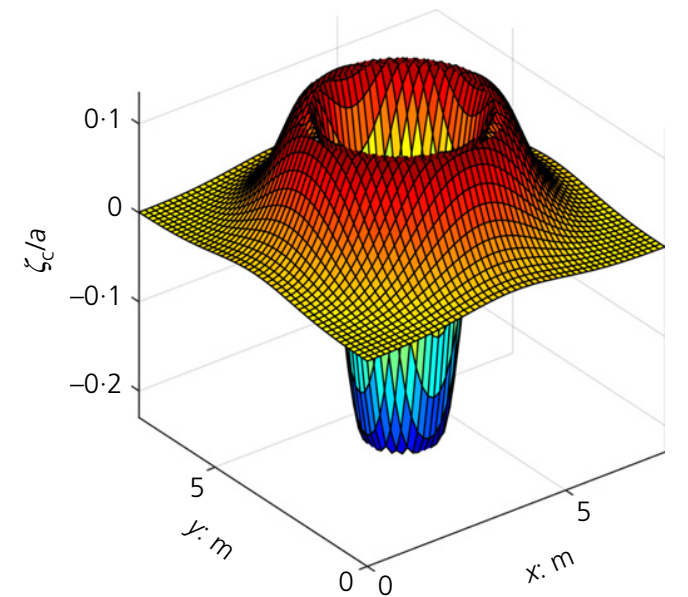

(a)

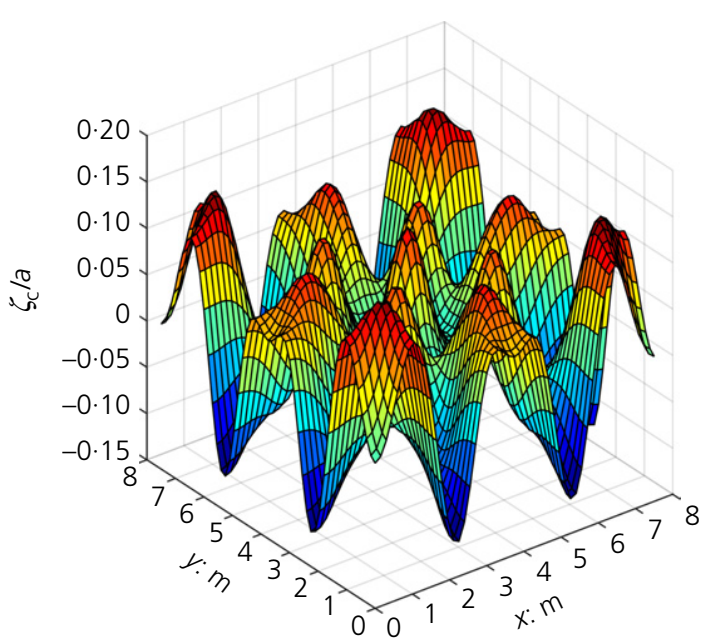

(c)

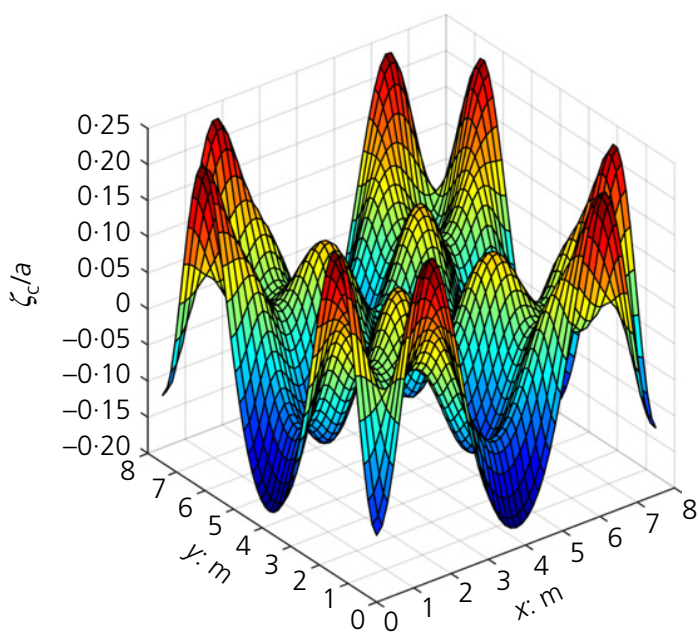

(b)

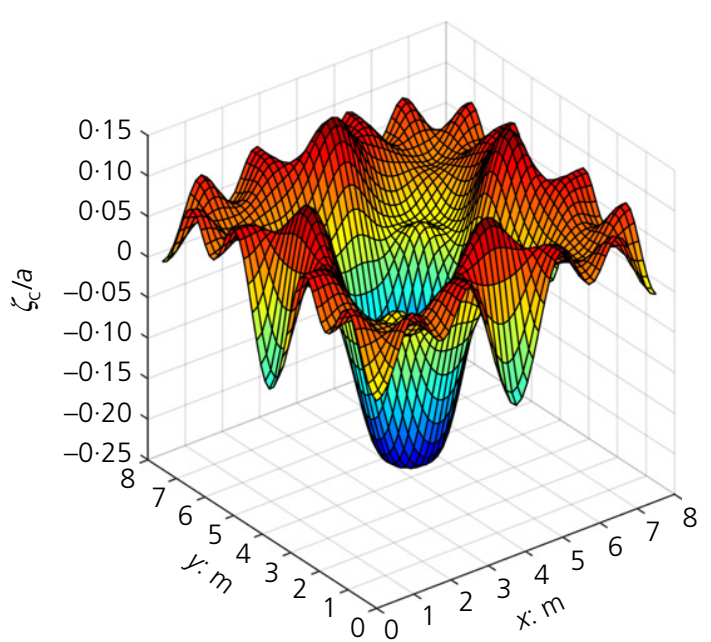

(d)

Figure 7. Analytical 3D visualisations of the sloshing of an initial Gaussian hump in a square, flat-bottomed basin: (a) $t=1 \mathrm{~s}$; (b) $t=5 \mathrm{~s}$; (c) $t=10 \mathrm{~s}$; and (d) $t=20 \mathrm{~s}$ 


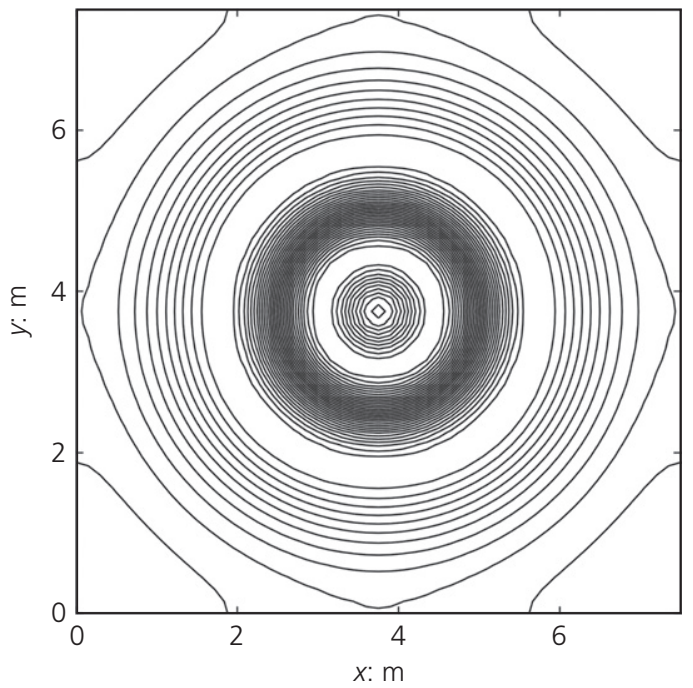

(a)

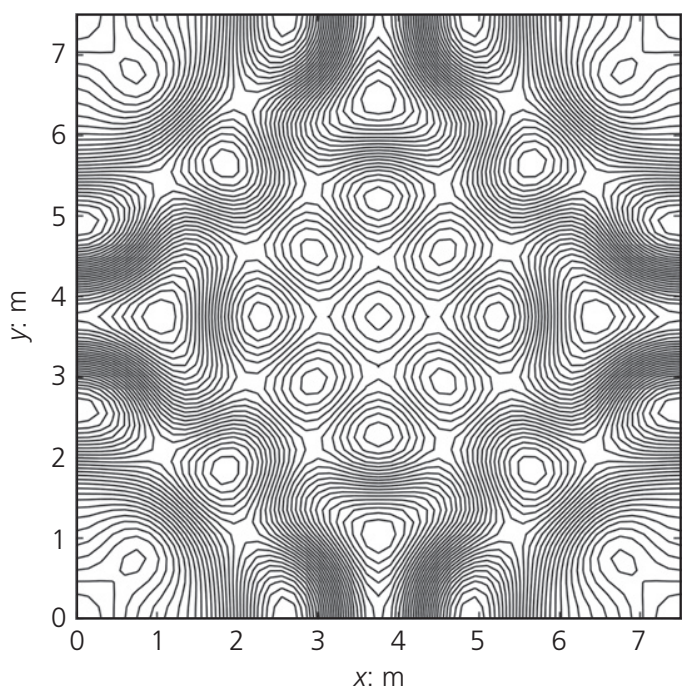

(c)

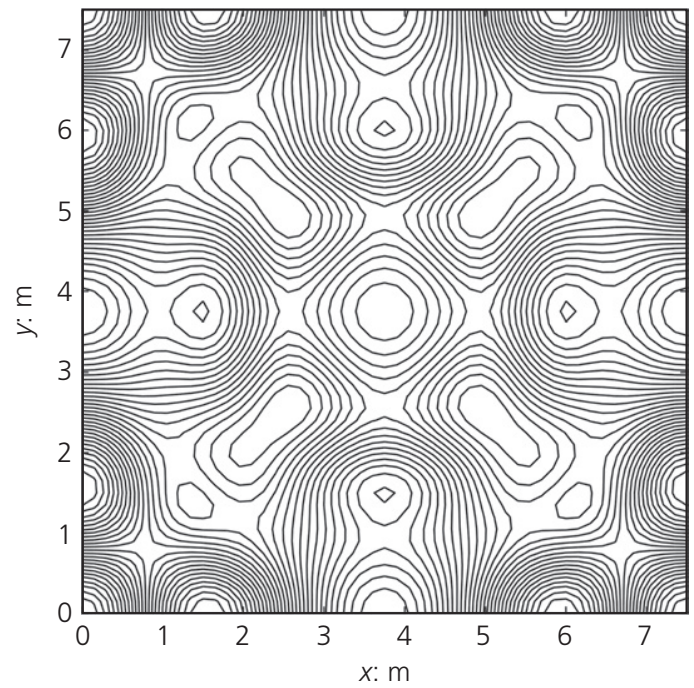

(b)

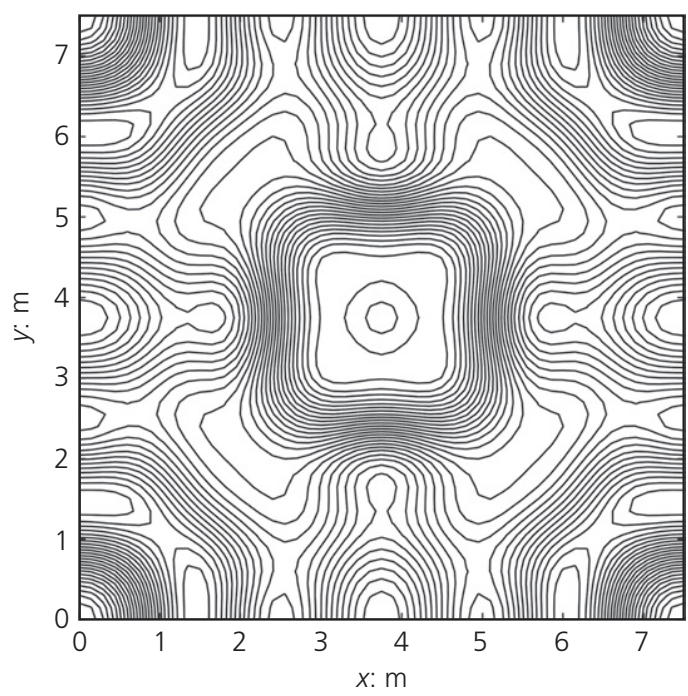

(d)

Figure 8. Predicted free surface contour maps of the sloshing of an initial Gaussian hump in a square, flat-bottomed basin: (a) $t=1 \mathrm{~s}$; (b) $t=5 \mathrm{~s}$; (c) $t=10 \mathrm{~s}$; and (d) $t=20 \mathrm{~s}$

the initial hump. Here, $\zeta_{\mathrm{c}} / a=1$ at time $t=0 \mathrm{~s}$ for free surface elevation of time history at the centre of the basin. There is close agreement between the numerical and analytical results for about $10 \mathrm{~s}$ after the initial hump is released, after which differences are discernible between the numerical predictions and analytical solution. The foregoing discrepancies are largely due to non-linear (second- and higher-order) wave interactions which are modelled by the 2D level I GN equations, but neglected in the analytical solution of the linearised SWEs. The even harmonics of the sloshing motions induced by the initial Gaussian hump can be determined by simulating the free surface time series resulting from releasing the initial hump, and the corresponding free surface time histories driven by an initial trough of equal but opposite shape to that of the hump (following the separation of harmonics method utilised by Borthwick et al. (2006), Hunt et al. (2004) and Johannessen and Swan (2001), among others). Here, the harmonics are treated as orthogonal functions, and the even harmonics obtained by addition as $\left(\zeta_{\mathrm{c}}+\zeta_{\mathrm{t}} / 2\right)$, where $\zeta_{\mathrm{c}}$ refers to the free surface elevation time series of the initial Gaussian hump and $\zeta_{\mathrm{t}}$ the equivalent time series for the initial Gaussian trough. Figure 4 shows that for a relatively large amplitude hump 
One-dimensional and two-dimensional Green-Naghdi equations for sloshing

Jalali and Borthwick

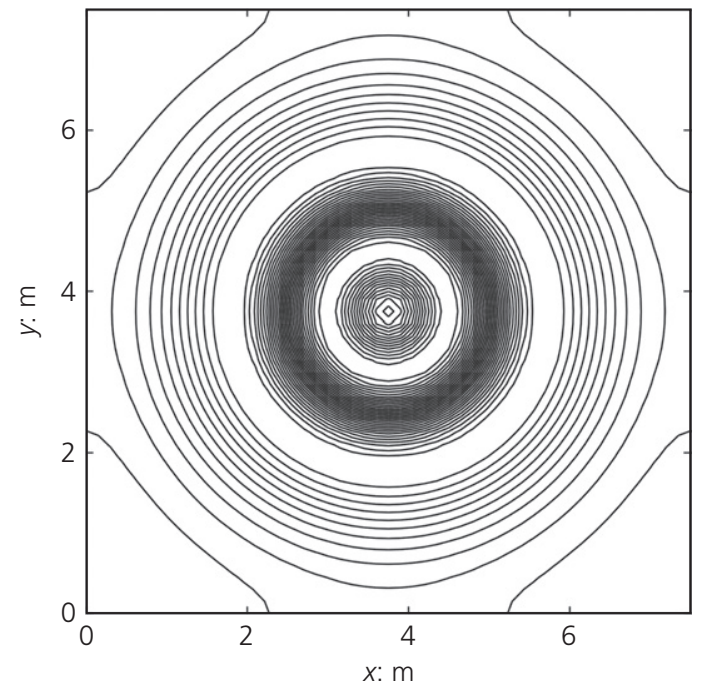

(a)

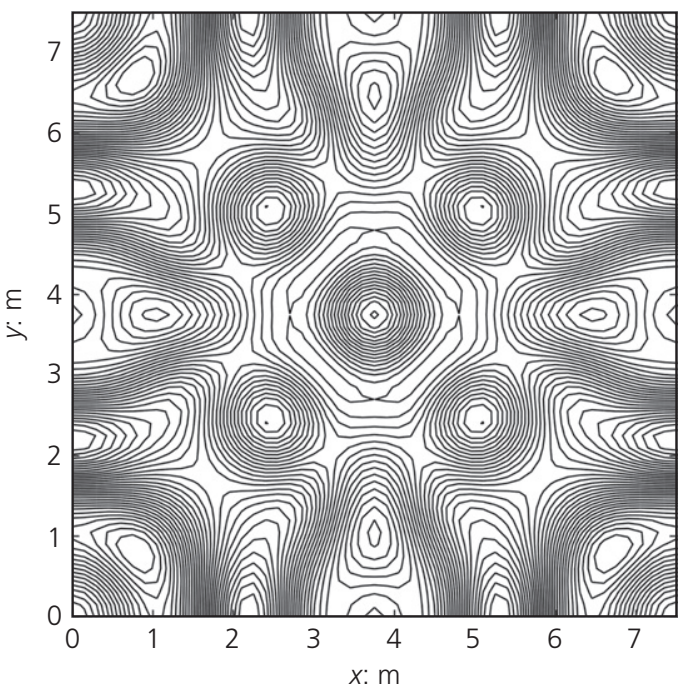

(c)

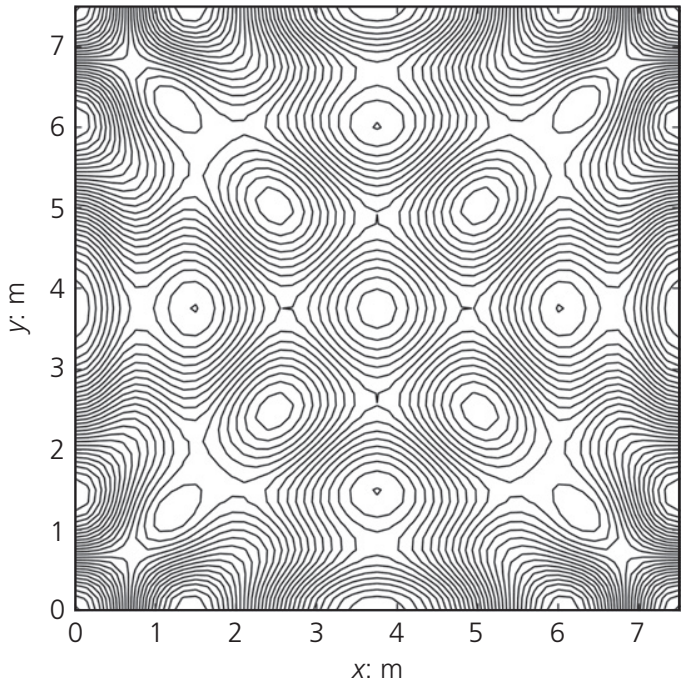

(b)

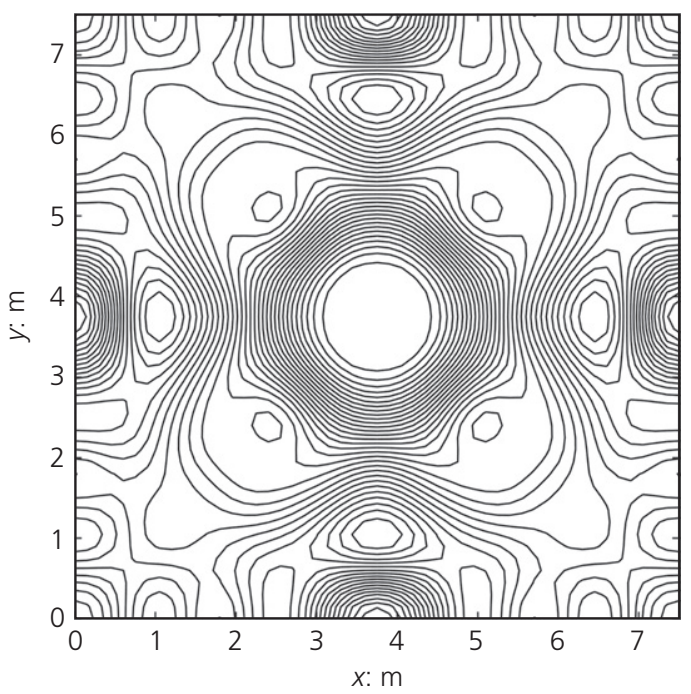

(d)

Figure 9. Analytical free surface contour maps of the sloshing of an initial Gaussian hump in a square, flat-bottomed basin: (a) $t=1 \mathrm{~s}$; (b) $t=5 \mathrm{~s}$; (c) $t=10 \mathrm{~s}$; and (d) $t=20 \mathrm{~s}$

$\left(a=h_{0} / 2=0 \cdot 225 \mathrm{~m}\right)$, it is possible to see evidence of the nonlinear effect produced by even harmonics, which are nondimensionalised with respect to the amplitude of the initial hump. The even harmonics have amplitudes of up to about $20 \%$ of that of the initial hump, and are perhaps growing slightly over the duration of the simulation. Figure 5 presents a comparison of the analytically predicted fast Fourier transform (FFT) spectrum for the free surface elevation time history of the initial Gaussian hump and the numerically predicted FFT spectrum for the free surface elevation time history of even harmonic components. It can be observed that all five peaks of numerical even harmonics occur at the same frequency as that of the analytically predicted peaks of Gaussian hump. Table 2 lists the resonant frequencies associated with different modes for the basin $(r$ and $s$ ): the first peak occurs at mode $r=2$ and $s=0$; the second peak at mode $r=2$ and $s=2$; the third peak at mode $r=3$ and $s=2$; the fourth peak at mode $r=4$ and $s=0$; and finally the fifth peak at mode $r=5$ and $s=1$.

Figures 6 and 7 compare the numerical simulations with the analytical solutions for sloshing in the basin, using 3D visualisations of the water surface at times $t=1,5,10$ and $20 \mathrm{~s}$. 
One-dimensional and two-dimensional Green-Naghdi equations for sloshing

Jalali and Borthwick

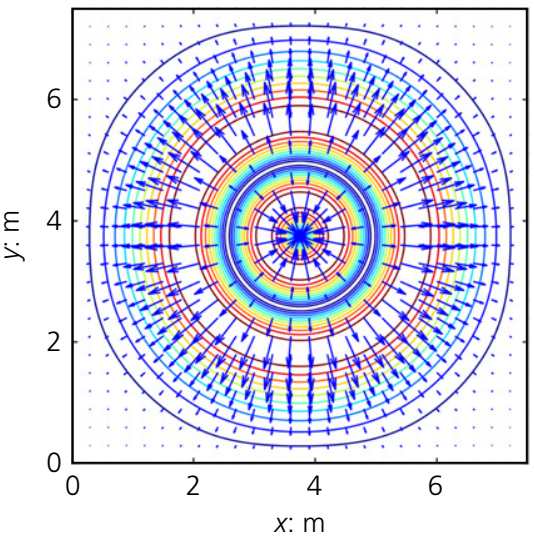

(a)

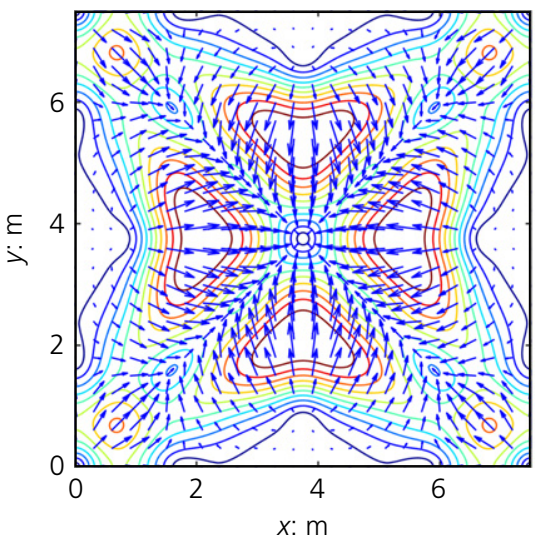

(c)
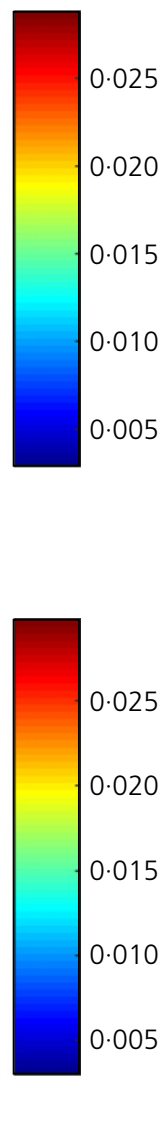

025

020

$\varepsilon$

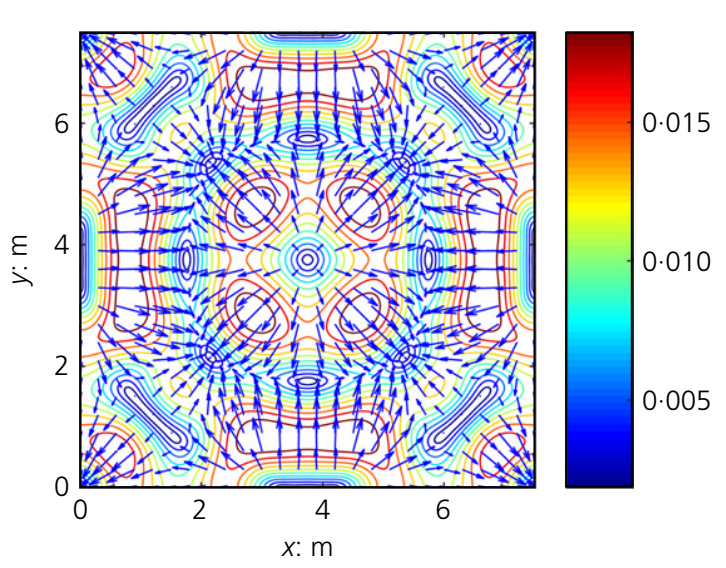

(d)

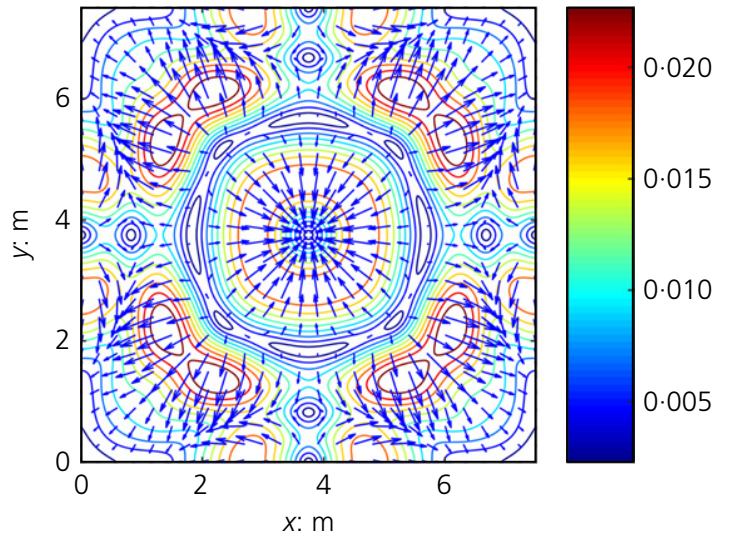

(b)

Figure 10. Numerically predicted velocity vectors and magnitude contours for the water surface in a square, flat-bottomed basin: (a) $t=1 \mathrm{~s}$; (b) $t=5 \mathrm{~s}$; (c) $t=10 \mathrm{~s}$; and (d) $t=20 \mathrm{~s}$

The corresponding contour maps are given in Figures 8 and 9. Although satisfactory agreement is achieved between the numerical predictions and analytical solution at $t=1$ and $5 \mathrm{~s}$, discrepancies between the numerical and analytical simulations become evident at $t=10 \mathrm{~s}$, and grow with simulation time, as can be seen at $t=20 \mathrm{~s}$ where phase differences are observable. Figure 10 shows the numerically predicted velocity vectors and magnitude contours for the water surface at times $t=1,5,10$ and $20 \mathrm{~s}$ after releasing the Gaussian hump in the flat-bottomed basin. Here, the velocity vectors indicate the direction of water particles and magnitude contours show the value of velocity in different sections of the basin. Slosh motions evolve in the basin from the initial hump as it rapidly drops under its own weight, causing a deep trough at the centre of the basin with an associated circular wavefront. The initial free surface motions are remarkably similar to those generated by the collapse of a liquid column, as modelled by Toro (2001), among others, except that the central oscillations do not die away as quickly. The balance between potential and kinetic energy drives repeated up and down motions at the centre of the basin, generating circular waves that propagate radially away from the centre of the basin and reflect with the basin walls. The repeated reflections between the waves with each other and the walls promote increasingly complicated sloshing modes dominated by waves whose wavelength is half the length of the basin.

Figure 11 compares long-time simulation of the numerical free surface elevation time history with the analytical solution at the centre of the basin for very small-amplitude disturbance $(a=0.001 \mathrm{~m})$ and spreading parameter $\left(b=0.2 \mathrm{~m}^{-2}\right)$. Here, a complete agreement between the numerical and analytical simulations is obtained since the effect of non-linear secondorder wave interactions is quite small. The corresponding FFT plots are given in Figure 12. The reversibility of the 


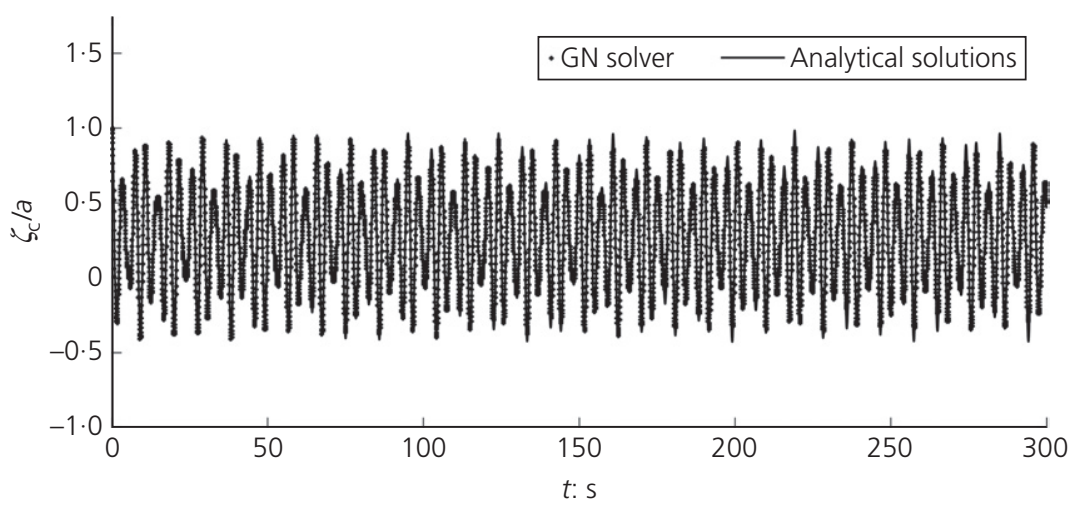

Figure 11. Analytical and predicted free surface elevation time histories at the centre of a basin for sloshing of an initial Gaussian hump in a square, flat-bottomed basin applying $a=0.001 \mathrm{~m}$ and $b=0.2 \mathrm{~m}^{-2}$

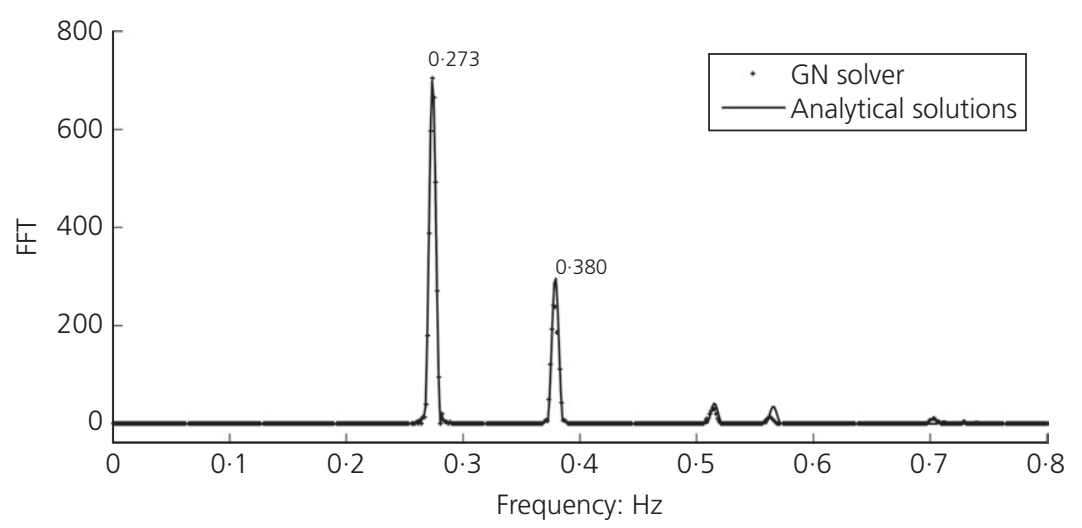

Figure 12. Comparison between analytically predicted FFT spectrum with the numerically predicted FFT spectrum for the free surface elevation time history of initial Gaussian hump applying $a=0.001 \mathrm{~m}$ and $b=0.2 \mathrm{~m}^{-2}$

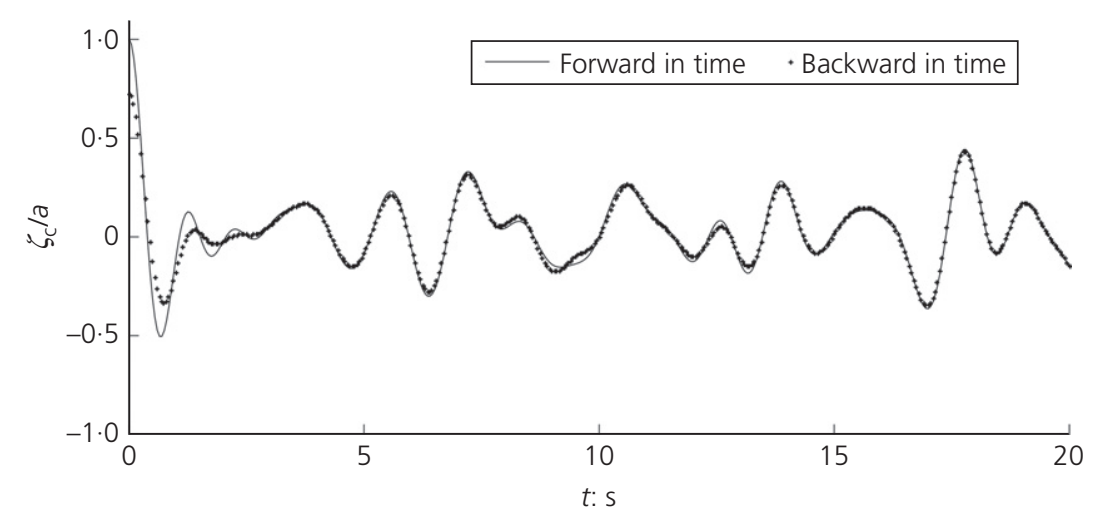

Figure 13. Reversibility test for Gaussian hump sloshing in a square, flat-bottomed basin: free surface elevation time history at the centre of basin for $a=0.045 \mathrm{~m}$ and $b=2 \mathrm{~m}^{-2}$ 
Engineering and Computational Mechanics Volume 170 Issue EM2
One-dimensional and two-dimensional

Green-Naghdi equations for sloshing

in shallow basins

Jalali and Borthwick

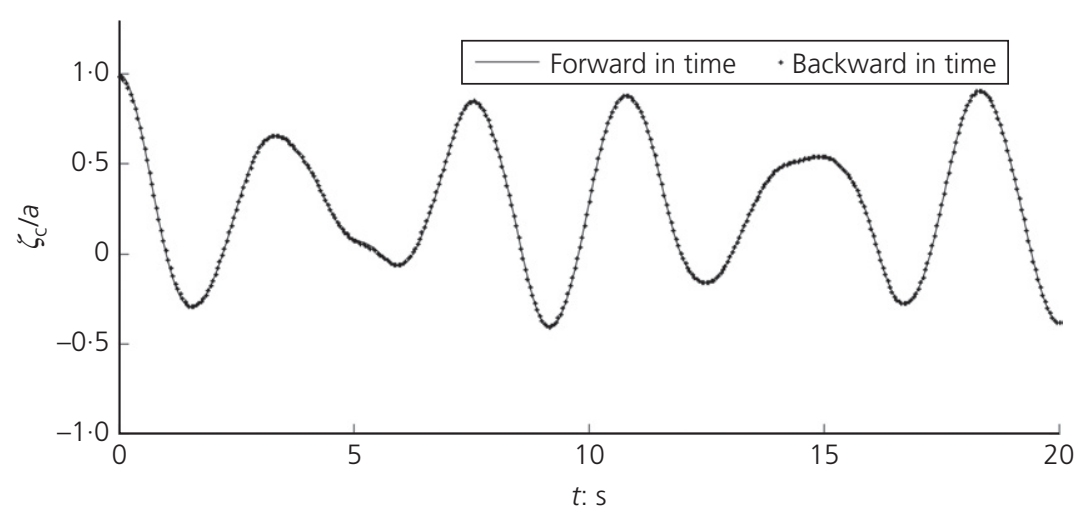

Figure 14. Reversibility test for Gaussian hump sloshing in a square, flat-bottomed basin: free surface elevation time history at the centre of basin for $a=0.001 \mathrm{~m}$ and $b=0.2 \mathrm{~m}^{-2}$

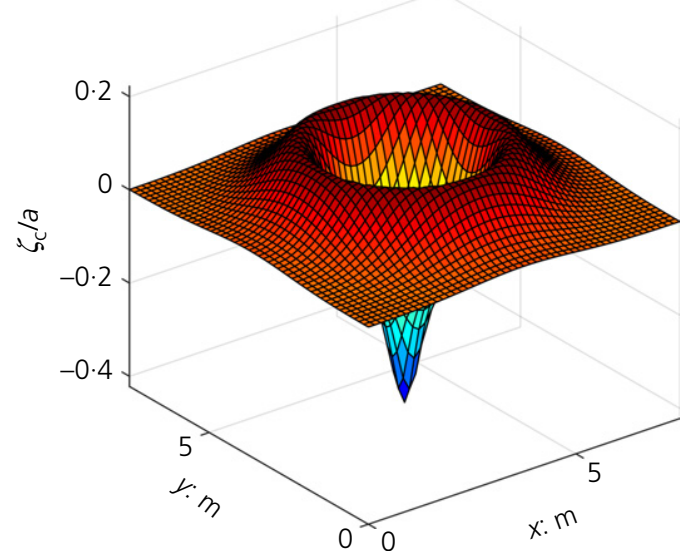

(a)

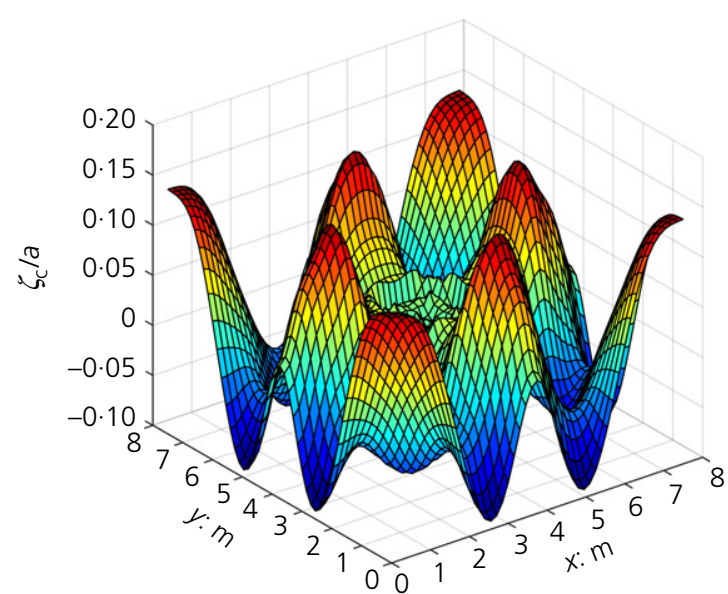

(c)

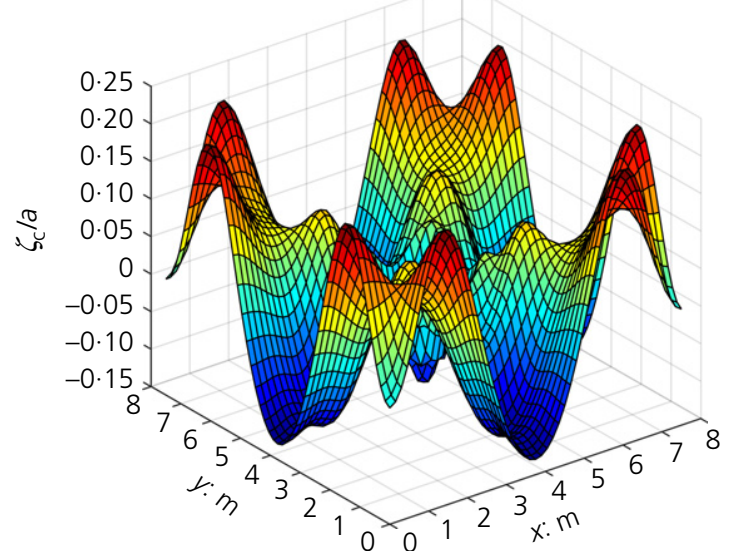

(b)

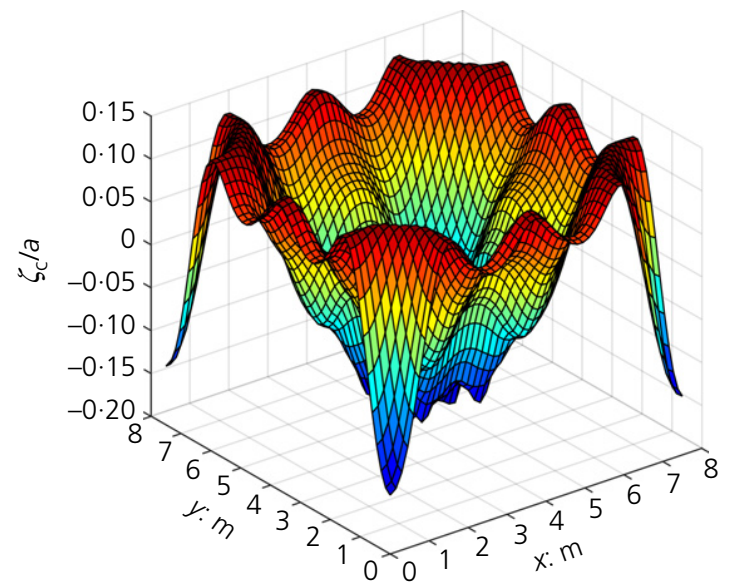

(d)

Figure 15. Predicted 3D visualisations of sloshing of an initial Gaussian hump in a square basin where the bed contains a central hump: (a) $t=1 \mathrm{~s}$; (b) $t=5 \mathrm{~s}$; (c) $t=10 \mathrm{~s}$; and (d) $t=20 \mathrm{~s}$ 


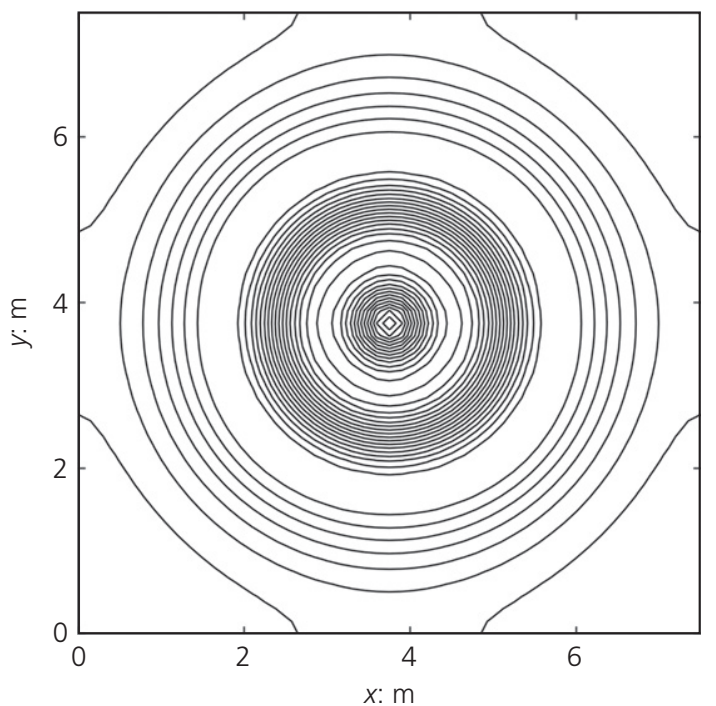

(a)

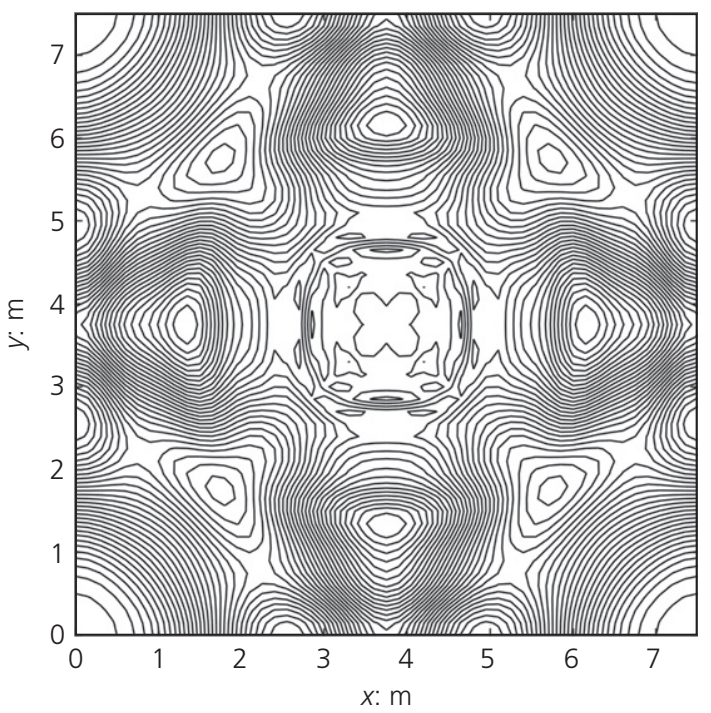

(c)

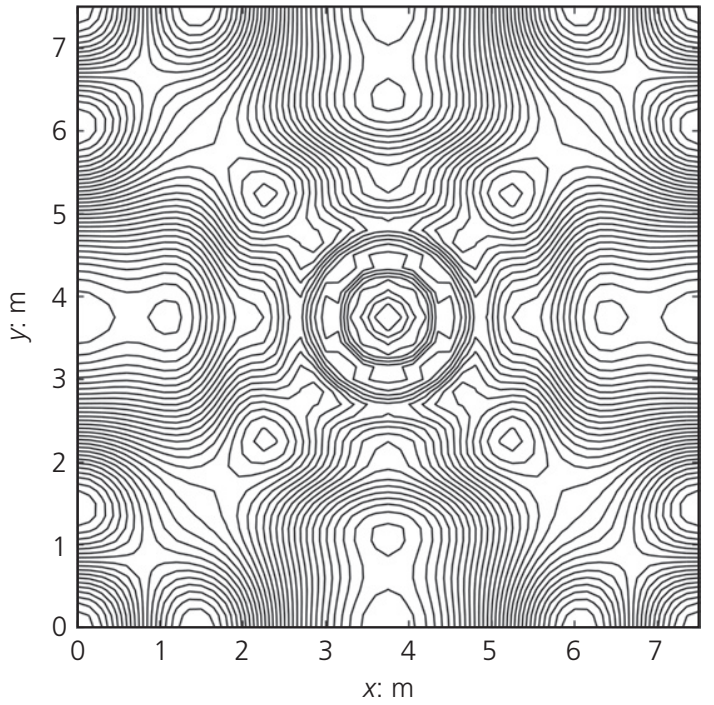

(b)

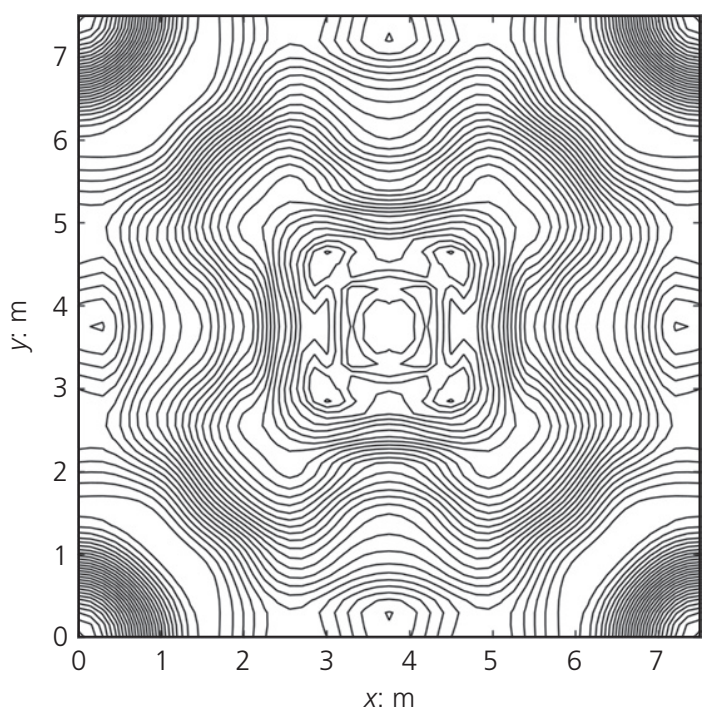

(d)

Figure 16. Predicted free surface elevation contour plots of sloshing of an initial Gaussian hump in a square basin where the bed contains a central hump: (a) $t=1 \mathrm{~s}$; (b) $t=5 \mathrm{~s}$; (c) $t=10 \mathrm{~s}$; and (d) $t=20 \mathrm{~s}$

simulations is now considered. The Gaussian hump is released at $t=0 \mathrm{~s}$ and the numerical solution then propagated forward in time until $20 \mathrm{~s}$, after which the time step is made negative and the numerical scheme is forced to simulate the backward propagation of the water surface until time zero is again reached. The results should be in almost identical agreement, given that the problem is thermodynamically reversible. There is no viscosity present, no turbulence, no surface tension and no sources of friction (e.g. from the basin walls or bed). Figure 13 examines reversibility by plotting the free surface elevation time history at the centre of the basin. The forward part of the simulation is shown by the solid line and the backward part by means of cross symbols. For the vast majority of the simulation, the agreement between the forward and backward processes is excellent. Discrepancies only occur when recovering the last $3 \mathrm{~s}$ of simulation. It seems likely that by travelling forward and backward in time, the accumulated dissipative error of the numerical scheme is responsible for causing the recovered hump to lose amplitude. Increasing the number of grids, increasing the number of iterations and selecting very 
One-dimensional and two-dimensional Green-Naghdi equations for sloshing

Jalali and Borthwick

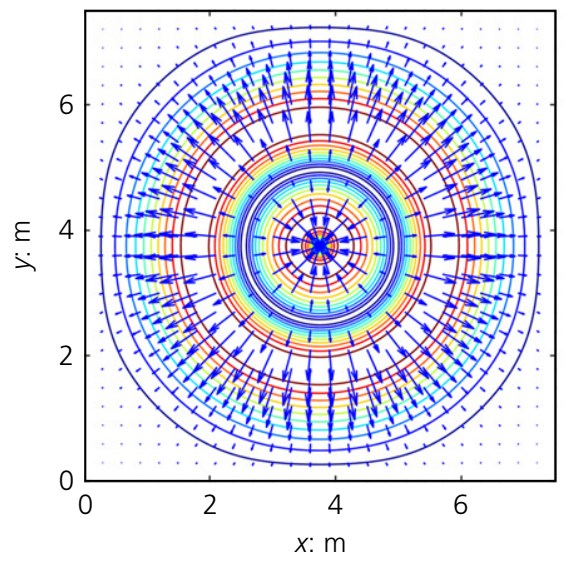

(a)

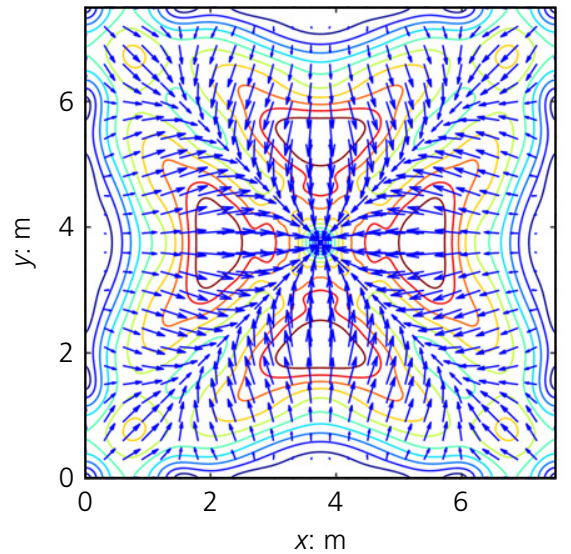

(c)
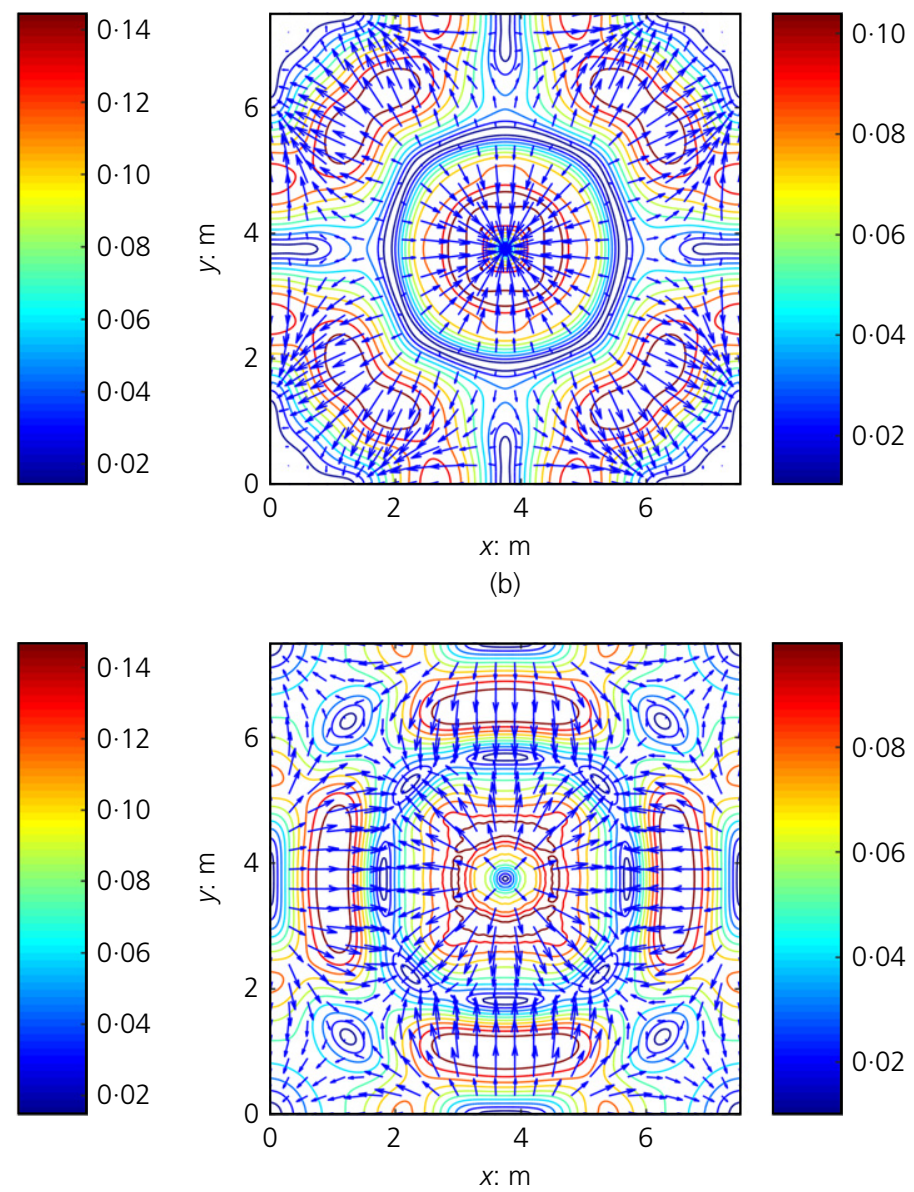

(d)

Figure 17. Predicted velocity vectors and magnitude contours for the water surface in a square basin where the bed topography contains a central hump: (a) $t=1 \mathrm{~s}$; (b) $t=5 \mathrm{~s}$; (c) $t=10 \mathrm{~s}$; and (d) $t=20 \mathrm{~s}$

small time steps did not reduce the magnitude of this accumulated dissipative error at the last $3 \mathrm{~s}$ of simulation. Figure 14 indicates the effect of reversibility on free surface elevation time history simulation at the centre of the basin for very small amplitude disturbance, $a=0.001 \mathrm{~m}$, and spreading parameter $b=0 \cdot 2 \mathrm{~m}^{-2}$. Excellent agreement is obtained between forward and backward propagations of the water surface since the effect of non-linearity is neglected.

\subsection{Parameter tests for sloshing in a square basin with non-uniform bathymetry}

Simulations are now considered of the sloshing behaviour of an initial Gaussian hump of water released in a square basin with non-uniform bathymetry. Here, the bed elevation is given by

33. $z_{\mathrm{b}}(x, y)=a_{z_{\mathrm{b}}} \exp \left\{-b_{z_{\mathrm{b}}}\left[\left(x-\frac{L_{x}}{2}\right)^{2}+\left(y-\frac{L_{y}}{2}\right)^{2}\right]\right\}$ where $a_{z_{\mathrm{b}}}$ is the bed amplitude and $b_{z_{\mathrm{b}}}=2 \mathrm{~m}^{-2}$ is a bed spreading parameter. The initial local free surface elevation is

34.

$$
\begin{aligned}
\zeta_{0}(x, y)= & -a_{z_{\mathrm{b}}} \exp \left\{-b_{z_{\mathrm{b}}}\left[\left(x-\frac{L_{x}}{2}\right)^{2}+\left(y-\frac{L_{y}}{2}\right)^{2}\right]\right\} \\
& +a \exp \left\{-b\left[\left(x-\frac{L_{x}}{2}\right)^{2}+\left(y-\frac{L_{y}}{2}\right)^{2}\right]\right\}
\end{aligned}
$$

where $a$ is the amplitude of the initial Gaussian hump in free surface elevation and $b=2 \mathrm{~m}^{-2}$ is a measure of its spread. Figures 15 and 16, respectively, depict 3D visualisation and contour maps of the water surface at times $t=1,5,10$ and $20 \mathrm{~s}$ for relatively large values of bed hump amplitude $\left(a_{z_{\mathrm{b}}}=0.225 \mathrm{~m}\right)$ and Gaussian hump amplitude $(a=0.225 \mathrm{~m})$. It is worth mentioning that the bed hump amplitude, $a_{z}$, is obtained through trial and error method (for more details see chapter 5 of the $\mathrm{PhD}$ thesis by Jalali (2016)). Figure 17 


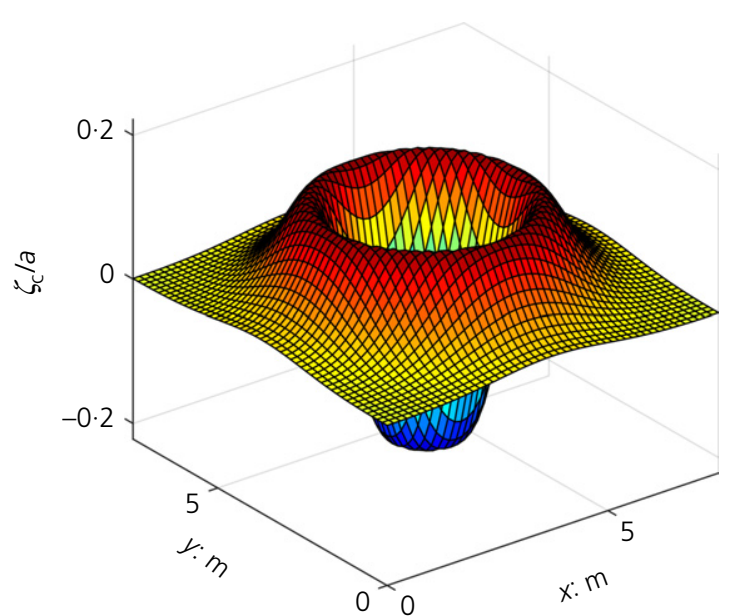

(a)

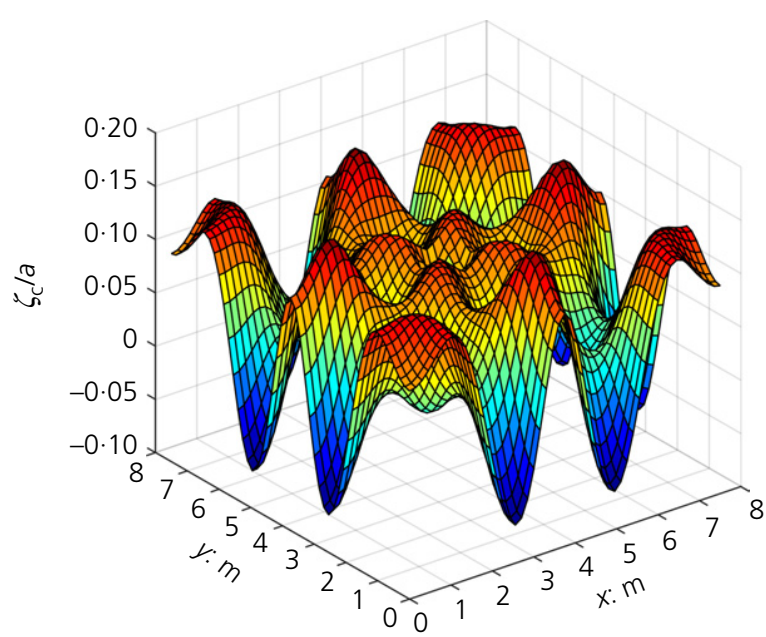

(c)

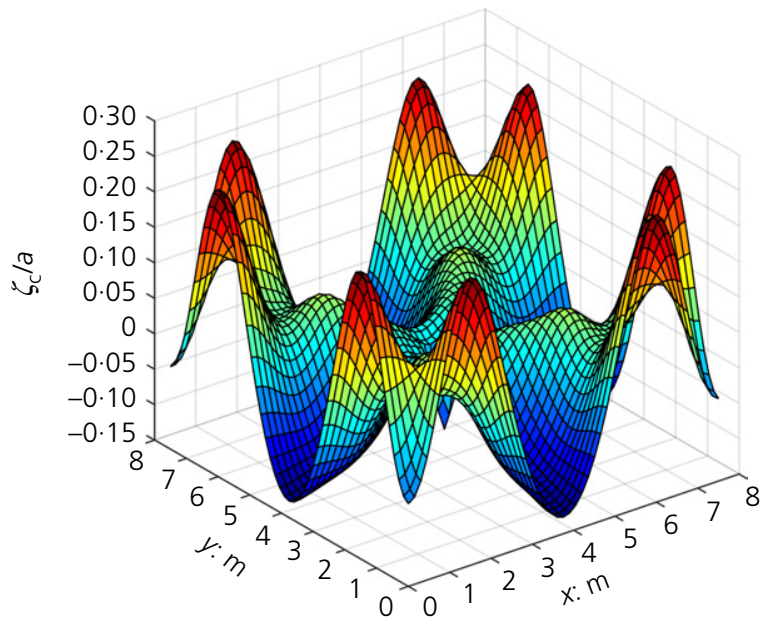

(b)

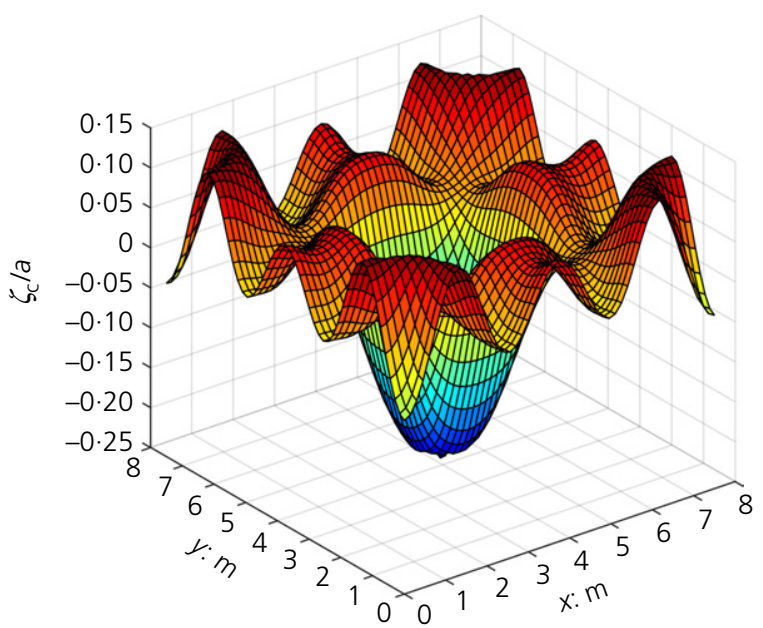

(d)

Figure 18. Predicted 3D visualisations of sloshing of an initial Gaussian hump in a square basin where the bed contains a central trough: (a) $t=1 \mathrm{~s}$; (b) $t=5 \mathrm{~s}$; (c) $t=10 \mathrm{~s}$; and (d) $t=20 \mathrm{~s}$

presents velocity vectors and magnitude contours for the water surface at times $t=1,5,10$ and $20 \mathrm{~s}$, where the bed topography contains a central hump. The effect of the bed hump on the evolution of the water free surface is most obvious at the centre of the basin exactly where the bed hump has its peak. At first, the Gaussian free surface hump drops rapidly to form a trough at the centre of the basin, releasing a circular ring-like wave that propagates towards the basin walls, where reflections occur. The plunging free surface at the centre of the basin interacts with the bed hump, leading to the recovery of a second clapotis-like hump, which peaks and releases a second circular wave. After several cycles of central peaks and troughs, the water surface motions immediately above the hump degenerate into a patch of small waves that heave up and down over the hump (after $t \sim 10 \mathrm{~s}$ ); elsewhere the sloshing behaviour is similar to that of the corresponding case without a bed hump, particularly the presence of sloshing components whose wavelength is half the length of the basin.

Figures 18 and 19, respectively, show 3D visualisation and contour maps of the evolution of the water surface over a Gaussian trough in the bed $\left(a_{z_{\mathrm{b}}}=-0.225 \mathrm{~m}\right)$. Figure 20 shows the velocity vectors and magnitude contours for the water surface at (a) $t=1 \mathrm{~s}$, (b) $t=5 \mathrm{~s}$, (c) $t=10 \mathrm{~s}$ and (d) $t=20 \mathrm{~s}$, where the bed topography contains a central trough. The bed trough has the greatest effect at the centre of the basin, coincident with the peak position of the initial Gaussian free surface 


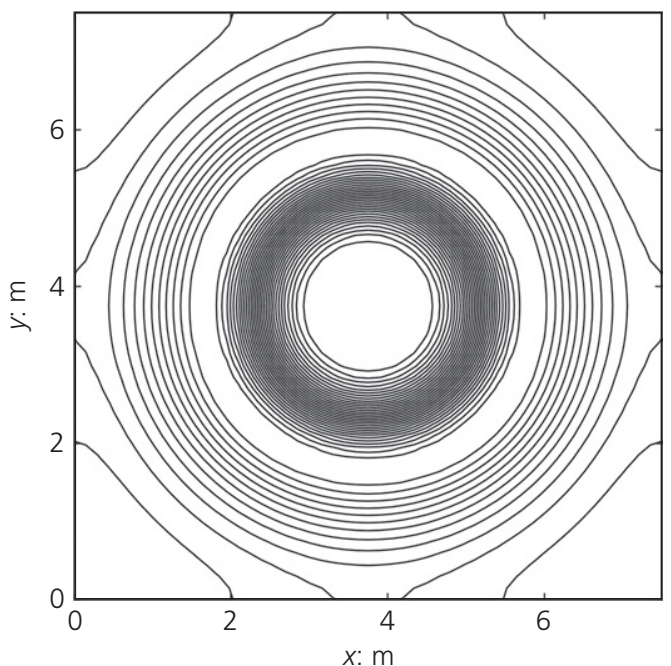

(a)

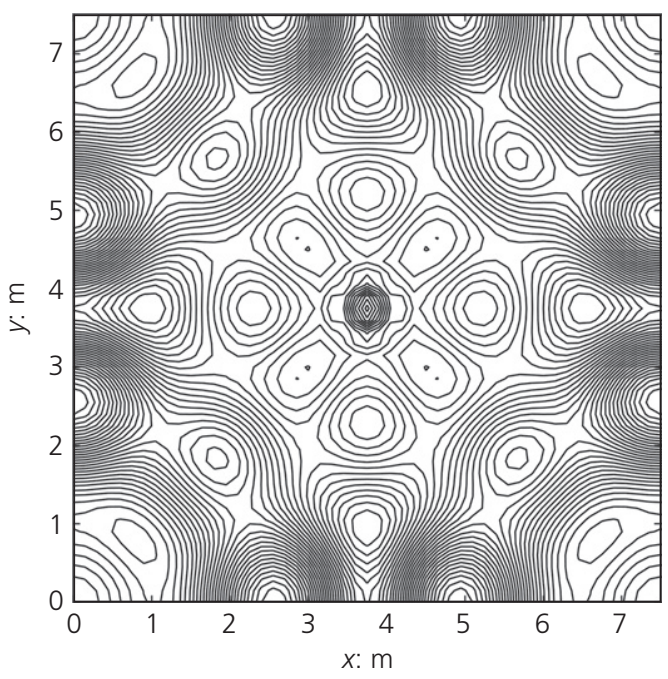

(c)

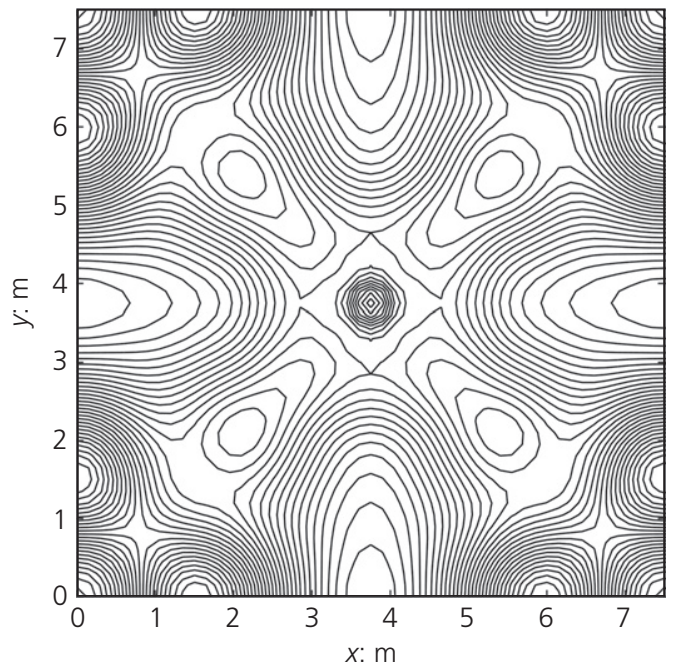

(b)

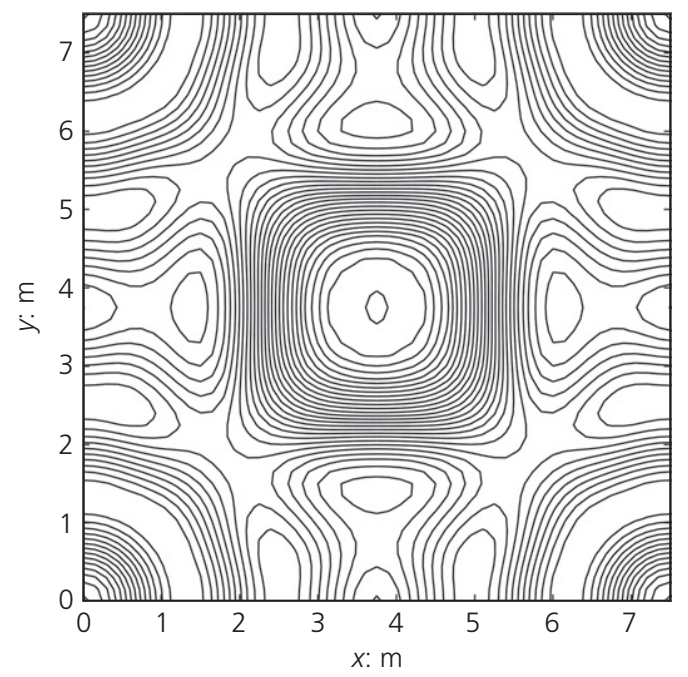

(d)

Figure 19. Predicted free surface elevation contour plots of sloshing of an initial Gaussian hump in a square basin where the bed contains a central trough: (a) $t=1 \mathrm{~s}$; (b) $t=5 \mathrm{~s}$; (c) $t=10 \mathrm{~s}$; and (d) $t=20 \mathrm{~s}$

hump. The water free surface at the centre of the basin is able to fall further than for the corresponding bed hump case, before interacting with the bed; localised sloshing of circular waves develops above the bed trough; the slosh behaviour away from the basin centre is similar to that in the corresponding basin with a flat bed, with modes at half basin wavelength dominating.

\section{Conclusions}

This study has presented level I GN equations for shallow flow over uniform and non-uniform bed topography in the context of slosh motions in a container. It has been demonstrated that level I GN equations can represent sloshing in a closed square basin resulting from initial sinusoidal and initial Gaussian free surface perturbations. Satisfactory agreement was obtained between the model predictions and the linear analytical solution for relatively small initial wave amplitude $(a \leq 0.005 \mathrm{~m})$. At larger amplitudes of initial disturbance, the numerically predicted free surface elevation time history steepened up and eventually began to develop a saw-tooth profile. Non-linear effects were particularly noticeable in the even harmonic slosh components. For $a=0 \cdot 045 \mathrm{~m}$ and $b=2 \mathrm{~m}^{-2}$ satisfactory agreement was also obtained between the numerical predictions and 


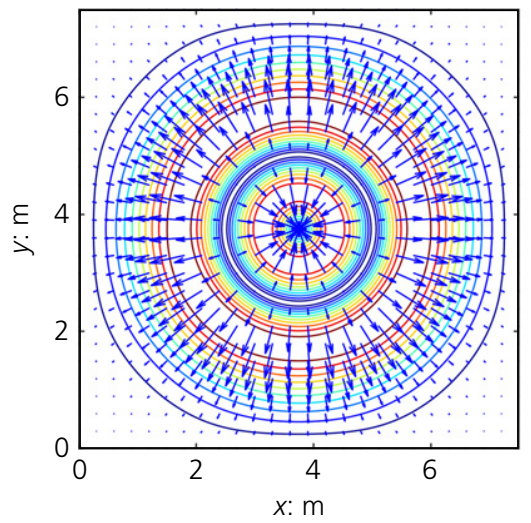

(a)

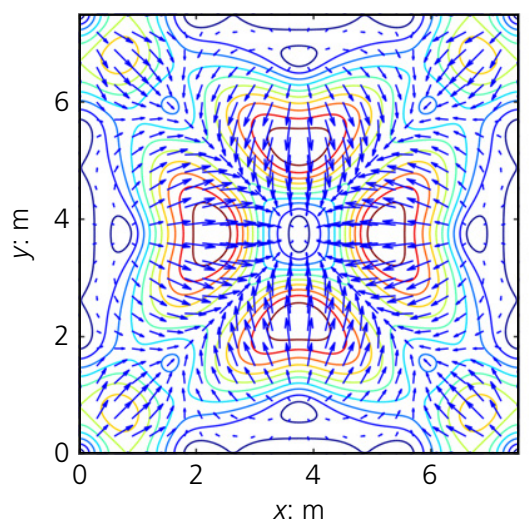

(c)
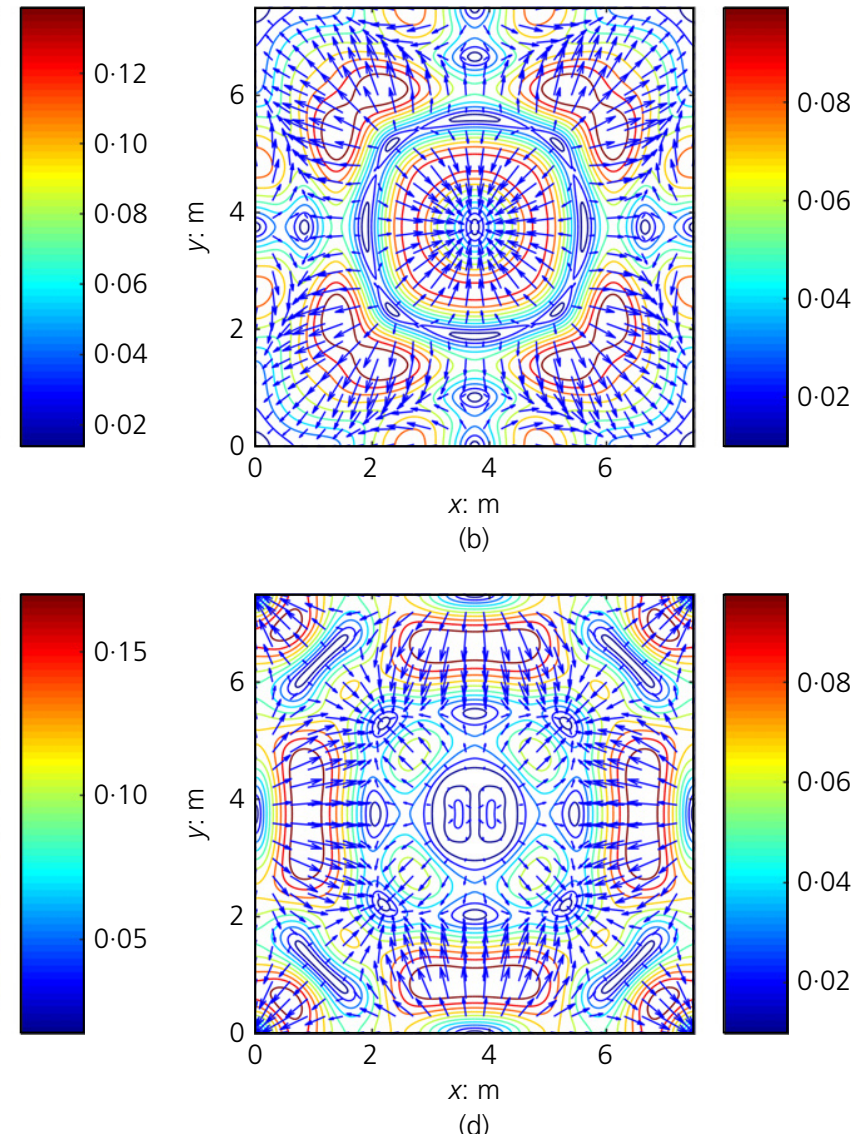

(d)

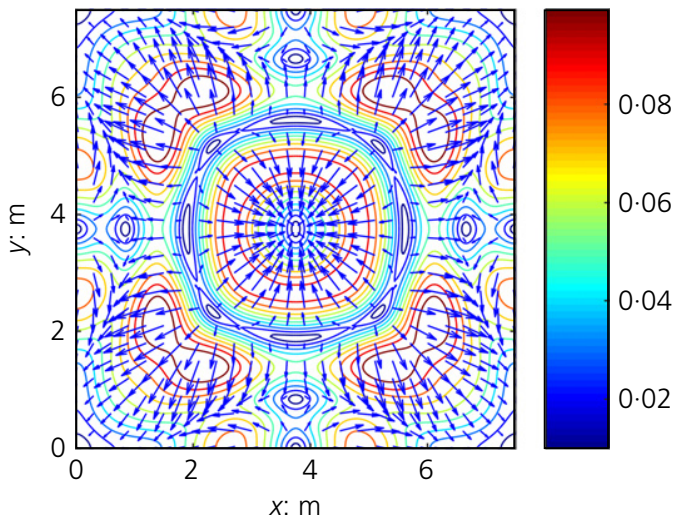

(b)

Figure 20. Predicted velocity vectors and magnitude contours for the water surface in a square basin where the bed topography contains a central trough: (a) $t=1 \mathrm{~s}$; (b) $t=5 \mathrm{~s}$; (c) $t=10 \mathrm{~s}$; and (d) $t=20 \mathrm{~s}$

semi-analytical solution of the early stages of free surface motions in a square, flat-bottomed basin after the initial release of the Gaussian hump. Discrepancies later evolved partly due to non-linear wave interaction effects, which were not described by the analytical theory. It was found that the non-uniform bathymetry has a localised effect on slosh motions.

\section{Acknowledgement}

The first author was partly funded by the University of Edinburgh.

\section{REFERENCES}

Bonneton P, Chazel F, Lannes D, Marche F and Tissier M (2011) A splitting approach for the fully nonlinear and weakly dispersive Green-Naghdi model. Journal of Computational Physics 230(4): 1479-1498.

Borthwick AGL, Hunt AC, Feng T, Taylor PH and Stansby PK (2006) Flow kinematics of focused wave groups on a plane beach in the UK Coastal Research Facility. Coastal Engineering 53(12): 1033-1044.
Dean RG and Dalrymple RA (2004) Modeling of beaches and shorelines. In Coastal Processes with Engineering Applications. Cambridge University Press, Cambridge, UK, pp. 301-342.

Demirbilek Z and Webster WC (1992) Application of the Green-Naghdi Theory of Fluid Sheets to Shallow-Water Wave Problems, Report 1. Model Development. US Army Engineers Waterways Experiment Station, Coastal Engineering Research Center, Washington, DC, USA, Technical Report CERC-92-11.

Green AE and Naghdi PM (1976) Directed fluid sheets. Proceedings of the Royal Society of London. Series A, Mathematical and Physical Sciences 347(1651): 447-473.

Haniffah MRM (2013) Wave Evolution on Gentle Slopes-Statistical Analysis and Green-Naghdi Modelling. PhD thesis, University of Oxford, Oxford, UK.

Hunt AC, Taylor PH, Borthwick AGL and Stansby PK (2004) Phase inversion and the identification of harmonic structure in coastal engineering experiments. In Proceedings of the ASCE 29th International Conference on Coastal Engineering, Lisbon, Portugal (Smith JM (ed.)). World Scientific, Hackensack, NJ, USA, pp. 1047-1059.

Ibrahim RA (2005) Liquid Sloshing Dynamics Theory and Applications. Cambridge University Press, Cambridge, UK.

Jalali MR (2016) One-Dimensional and Two-Dimensional Green-Naghdi Equation Solvers for Shallow Flow Over Uniform 
and Non-Uniform Beds. PhD thesis, The University of Edinburgh, Edinburgh, UK.

Johannessen T and Swan C (2001) A laboratory study of the focusing of transient and directionally spread surface water waves. Proceedings of the Royal Society of London. Series A, Mathematical, Physical and Engineering Sciences 457(2008): 971-1006.

Lamb H (1916) Hydrodynamics. Tidal Waves, 4th edn. Cambridge University Press, Cambridge, UK.

Nadiga BT, Margolin LG and Smolarkiewicz PK (1996) Different approximations of shallow fluid flow over an obstacle. Physics of Fluids 8(8): 2066-2077.

Press WH, Teukolsky SA, Vetterling WT and Flannery BP (2007) Numerical Recipes the Art of Scientific Computing. Solution of Linear Algebraic Equations. Cambridge University Press, Cambridge, UK. Sarpkaya T and Isaacson M (1981) Mechanics of Wave Forces on Offshore Structures. Van Nostrand Reinhold, New York, NY, USA.
Shields JJ and Webster WC (1988) On direct methods in water-wave theory. Journal of Fluid Mechanics 197: $171-199$.

Toro EF (2001) Shock-Capturing Methods for Free-Surface Shallow Flows. John Wiley and Sons Ltd, Chichester, UK.

Webster WC and Shields JJ (1991) Applications of high-level Green-Naghdi theory to fluid flow problems. In Dynamics of Marine Vehicles and Structures in Waves (Price WG, Temarel P and Keane AJ (eds)). Elsevier Science, Amsterdam, the Netherlands, pp. 109-124.

Wei G and Kirby JT (1995) Time-dependent numerical code for extended Boussinesq equations. Journal of Waterway, Port, Coastal, and Engineering 121(5): 251-261.

Yao Y (2007) Boussinesq-Type Modelling of Gently Shoaling Extreme Ocean Waves. PhD thesis, University of Oxford, Oxford, UK.

\section{How can you contribute?}

To discuss this paper, please email up to 500 words to the editor at journals@ice.org.uk. Your contribution will be forwarded to the author(s) for a reply and, if considered appropriate by the editorial board, it will be published as discussion in a future issue of the journal.

Proceedings journals rely entirely on contributions from the civil engineering profession (and allied disciplines). Information about how to submit your paper online is available at www.icevirtuallibrary.com/page/authors, where you will also find detailed author guidelines. 